\title{
LE MERCURE PHALLIQUE DU MAS-D'AGENAIS ET UN DIEU STYLITE INÉDIT : Curiosités ou ("chaînons manquants )?
}

\author{
par Jacques SANTROT
}

Déjà connu par les travaux d'Alexandre Nicolä̈', le site gallo-romain du Mas-d'Agenais (Lot-etGaronne $)^{2}$ n'a cessé de livrer un mobilier important auquel P. Cadenat a consacré de nombreuses publications $^{3}$. C'est en 1983 que, lors d'une prospection de surface, J.-P. Noldin découvrit sur le site ${ }^{4}$ une pendeloque figurée en bronze moulé (H. : $3,3 \mathrm{~cm} ; 1 .: 2,5 \mathrm{~cm}$; ép. : $1,5 \mathrm{~cm}$; poids : $22,1 \mathrm{~g})^{5}$.

1 A. Nicolaï, Le Mas-d'Agenais sous la domination romaine et le cimetière gallo-romain de Saint-Martin, Bordeaux, Ferret éd., 1896. Id., Le cimetière gallo-romain de Saint-Martinde-Lesque du $I^{\mathrm{er}}$ au $I I I^{\mathrm{e}} \mathrm{s}$., dans Bull. du Comité des Travaux Historiques, 1897, p. 84-95. Id., La nécropole gallo-romaine de Saint-Martin-de-Lesque (Ussubium), dans Revue de l'Agenais, 55,1928 , p. 3-16.

2 A $15 \mathrm{~km}$ au sud-est de Marmande (Lot-et-Garonne), le site du Mas-d'Agenais domine la vallée de la Garonne (fig. 11, cf. note 1). Il a été identiflé comme la nécropole d'une agglomération dont on n'a pas encore retrouvé les vestiges. Pour être traditionnelle, cette attribution nous paraît sujette à caution. En effet, si la vingtaine de fosses fouillées renfermaient un mobilier important et très divers, avec une faune très abondante (vestiges de reliefs de cuisine mais, peut-être aussi, d'offrandes), on n'a jamais découvert sur le site d'urne cinéraire, caractérisée par un important dépôt d'esquilles osseuses humaines, ni le moindre os humain, "pas mêne une dent".

A titre de comparaison, les urnes cinéraires recueillies dans la nécropole de Terre-Nègre, à Bordeaux (Gironde), renfermaient un à deux litres d'esquilles osseuses, brùlées mais incompressibles, et de cendres. D'après leur description, les fosses observées au Mas-d'Agenais seraient plutôt pour les unes, de simples dépotoirs, pour d'autres des dépôts d'offrandes, les témoignages probables d'un culte indigène, chthonien, peut-être funéraire, comme il s'en rencontre fréquemment dans le Sud-Ouest sans qu'ils soient toujours bien identifiés (à Saintes (Charente-Maritime), aux Allées de Tourny et à la Place de la République de Bordeaux, en particulier). Ce n'est pas une nécropole à proprement parler puisque l'on n'a trouvé aucun vestige osseux humain.
La relative abondance des témoignages du travail de l'os et des métaux, des ratés de cuisson et des colifichets provenant d'ateliers de potiers, montre que l'on est aussi dans une zone de dépotoirs, à la périphérie immédiate de l'agglomération. C'est également le cas des puits "funéraires" et des fosses "rituelles " trouvées à Saintes (Ateliers Municipaux, Petites Sœurs des Pauvres) ou à Bordeaux (Allées de Tourny, Place de la République).

Identifé comme l'antique Ussubium par l'inscription C.I.L., XIII, 919, Le Mas-d'Agenais correspond à la station Ussubium, Usubium, Vassubium ou Vesubium (selon les manuscrits) que l'Itinéraire d'Antonin, et la Table de Peutinger placent sur la voie de Bordeaux à Agen, entre Sirio (Cérons) et Fines (localité indéterminée). Cf. C. Jullian, Inscriptions romaines de Bordeaux, II, 1890, p. 220-223, et P. CAdENAT, Nouvelles recherches dans la nécropole gallo-romaine d'VSSVBIVM (dite aussi, de Saint-Martin), commune du Masd'Agenais, 1975, Société Académique d'Agen, 1982, p. 9-10, et bibliographie p. 209-210.

3 En dernier lieu, la synthèse de P. Cadenat : Nouvelles recherches..., op. cit.

4 Le Mas-d'Agenais (Lot-et-Garonne), extrait de la section $\mathrm{ZB}$, parcelle no $39 \mathrm{du}$ cadastre. Cette parcelle, sur laquelle il n'a pas été trouvé de fosse, jouxte la parcelle no 42 où quatorze fosses furent fouillées. Ces deux parcelles constituent le lieu-dit "La Gaule et le Chemin du Milieu" (sic).

5 Collection J.-P. Noldin, Miramont-de-Guyenne (Lotet-Garonne). Le Mercure phallique du Mas-d'Agenais est déposé au Musée du Groupe Archéologique de Sainte-Bazeille (Lot-et-Garonne). Nous remercions très chaleureusement M. Jean-Paul Noldin de nous avoir communiqué cette œuvre et d'avoir bien voulu nous autoriser à la publier. 


\section{I - Le Mercurf phallique du Mas-d'Agenais}

\section{Description}

Il s'agit du dieu Mercure, nu, en buste coupé à mi-épaule. Lc cou est fort, presque cylindrique, ct la tête est légèrement tournée vers la droite. Le visage est ovale, aux pommettes saillantes et aux joues pleines. Le menton est peu marqué. Sous des arcades sourcilières puissantes, les yeux sont figurés par des globules sans pupille. Très sommairement indiquée, la bouche esquisse un timide sourire, avec deux profondes fossettes aux commissures des lèvres ${ }^{6}$ (fig. 1).

Sur le front, à la naissance des cheveux, sont figurés des organes génitaux masculins en érection. De part et d'autres, sur les tempes, prennent naissance deux ailerons, aux rémiges précisées par incision au burin, entre lesquels a été aménagée une importante bélière transversale, discoïdale, dont l'usure montre qu'elle a longtemps servi à la suspension de l'objet.

Plaquée par un fin galon qui court du sommet du crâne à la nuque, la chevelure est traitée en calotte hémisphérique aux mèches rayonnantes, à peine perceptibles. Des touffes courtes et raides s'échappent sur la nuque alors que trois boucles en fort relicf garnissent chaque tempe, entre l'aileron et l'oreille.

Le visage ovale ne manque pas de finesse. Le modelé est souple et suggère avec bonheur la musculature du buste divin.

La pièce est complète et en bon état de conservation. Elle présente une belle patine vert clair, à peu près régulière, provenant d'une couche de corrosion très pulvérulente à la découverte?

\section{Technique et origine de fabrication}

Le Mercure du Mas-d'Agenais semble avoir été fabriqué selon la technique de la fonte à la cire perdue. L'objet moulé a été retouché à la lime (facettes sur le cou) et au burin (retouches de la chevelure).

Les dimensions très réduites de cette pendeloque la rendent difficile à classer. Cependant, les proportions de la tête ne sont pas inhabituelles dans la petite statuaire votive : ce sont celles d'une statuette de 12 à $13 \mathrm{~cm}$ de hauteur dont on aurait tronçonné le torse.

A notre connaissance, le Mercure phallique du Mas-d'Agenais est une pièce unique dans l'iconographic du dieu, sans parallèle stylistique utile avec un bronze aquitain, et, faute de connaître la composition du métal, il n'est pas possible actuellement d'en préciser le lieu de fabrication. On sait aujourd'hui qu'une origine aquitaine est techniquement possible ${ }^{8}$ alors que, naguère encore, tout bronze au traitement un peu raffiné était qualifié de "travail italien ». Cependant, la référence aux lintinnabula et autres amulettes d'origine italienne est ici évidente.

\section{Destination de l'objet}

La taille $(\mathrm{H} .: 3,3 \mathrm{~cm})$ el le très faible poids de cet objet $(22,1 \mathrm{~g})$ excluent son utilisation comme

6 Le "sourire" n'est pas fréquent dans les figuralions divines gallo-romaines, pas plus que dans la statuaire grécoromaine depuis la disparition du "sourire archaïque ". Leur visage cost sévère, le plus souvent, ou simplement inexpressif. La bienveillance du petit Mercure de Monbazillac (Dordogne) est inhabituelle : Musée d'Aquitaine, Bordeaux, inv. 79.16.1. (fi. J. Saxtrot et D. Frugirn, dans Gallia, 39, 1981, 2, p. $211-217$

7 Nettoyage, restauration el stabilisation par $\mathbf{M}^{\text {me }}$ Brigitte Dr:kıx, Conservaleur au Musée d'Aquilaine de la Ville de
Bordeaux, chargée du Laboratoire Technique de Reslauration. 8 Г. Веск, М. Mexu, Th. Benthoud et I..-P. Huntei, Mélallurgie des bronzes, dans Recherches gallo-romaines I du Laboratoire de Recherche des Musées de France, collection "Notes et Documents " de la Réunion des Musées Nationaux, Paris, R.M.N., 1985, p. 71-142, passim. Il résulte notamment de cette étude que le célèbre Hercule de Bordeaux, statue "plus grande que nature ", est un "bronze au plomb homogène, caracléristique de la production d'Aquilaine", (p. 91). 

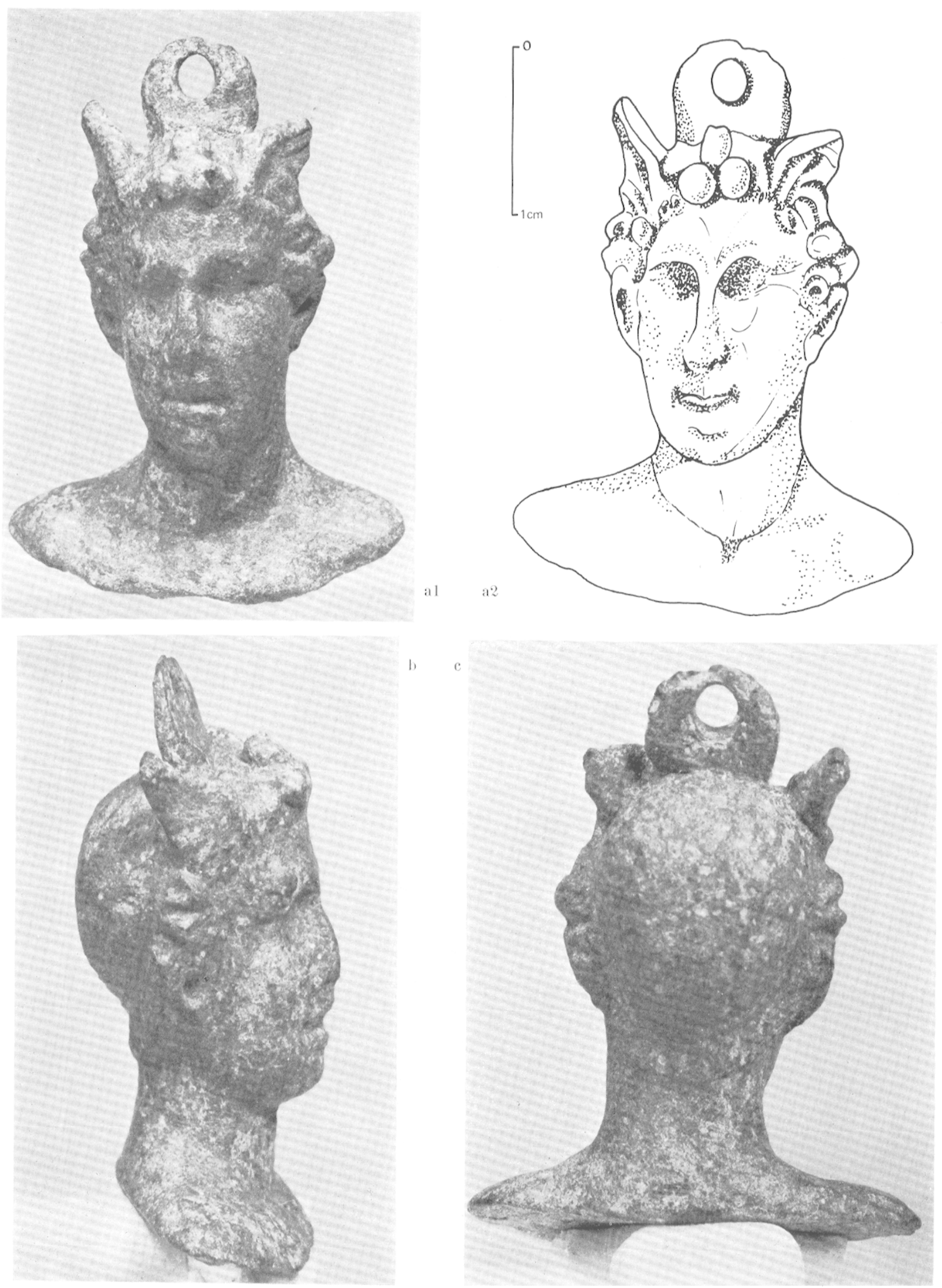

l Mercure phallique du Mas-d'Agenais. (H. : $3,3 \mathrm{~cm}$ ). al et a2 : face; $\mathrm{b}:$ profil à droile ; $c$ : los. 
"peson » ou "poids-curseur» de balance à fléau (aequipondium) 9 . Plus qu'un accessoire de divinité panthée ${ }^{10}$, il faut probablement voir dans ce petit buste à bélière l'un de ces talismans parmi lesquels les amulettes phalliques ou même triphalliques sont nombreuses ${ }^{11}$.

\section{Date de fabrication}

Le Mercure phallique du Mas-d'Agenais a été trouvé en surface. Son contexte archéologique ne permet guère d'en préciser la chronologie, si ce n'est par la durée proposée pour l'occupation du site : le mobilier recueilli dans les fosses du plateau Saint-Martin date de la période comprise entre le début du I $^{\text {er }}$ siècle ap. J.-G. et le dernier quart du III $^{\mathrm{e}}$ siècle, et même le début du $\mathrm{IV}^{\mathrm{e}}{ }^{\mathrm{e}}$.

Les faibles dimensions de cet objet rendent fort difficile une analyse stylistique dont les arguments ne peuvent qu'être aléatoires. De l'absence du traitement de l'iris, nous ne tirerons pas de conclusions. En revanche, le port du cordon plaquant la chevelure rappelle la coiffure du Jupiter dit "de Bree », une œuvre «de style sévère et éclectique » que l'on a datée de l'époque d'Hadrien, voire de la seconde moitié $d u \mathrm{II}^{\mathrm{e}}$ siècle ${ }^{13}$, mais ce même accessoire est porté par un Lare de Vienne attribué au r ${ }^{\mathrm{er}}$ siècle ${ }^{14}$. Le traitement de la chevelure, sur les tempes, peut être comparé à celui des boucles relevées et foisonnantes du Mercure assis de Saint-Marcel (Creuse) ${ }^{15}$. Cette statuctte dont la bouche un peu mièvre, aux commissures creusées, évoque celle du dieu du Mas-d'Agenais, a été trouvée dans un contexte daté entre le début du II ${ }^{\mathrm{e}}$ siècle et le troisième quart du III ${ }^{\mathrm{e}}$ siècle. La forme du visage et la disposition des boucles temporales rappellent également le beau Mercure de Thiennes (Nord) $\left(\mathrm{I}^{\mathrm{er}}-\mathrm{III}^{\mathrm{e}} \mathrm{s} .\right)^{16}$.

Comme on le voit, les comparaisons stylistiques sont minces. Elles offrent une datation large les trois premiers siècles de notre ère - qui est en accord avec le contexte archéologique. En se fondant sur la coiffure, il nous parait ccpendant légitime de préférer le II $^{\mathrm{e}}$ siècle, au plus tôt, pour situcr la date de fabrication de cette amulette ${ }^{17}$. Cette hypothèse est également en accord avec la chronologie des figurations phalliques, surtout fréquentes au $\mathrm{II}^{\mathrm{e}}$ et au $\mathrm{III}^{\mathrm{e}}$ siècle.

9 Les bronziers antiques ont souvent donné la forme de bustes de divinités ou de personnages divers aux aequipondia ou contrepoids de balances à fléau. Par exemple : Germaine FAIDER-Feytmans, Les bronzes romains de Belgique, Mayence, $1979, n^{\text {os }} 248$ à 250 : buste bacchique, aurige, tête masculine. P. LEBEL et Stéphanie Boccher, Bronzes figurés anliques du Musée Rolin d'Aulun, 1975, n 91 : buste de Silène. Stéphanie Boucher, G. Perdu, M. Feugtrre, Bronzes antiques du Musée de la Civilisation gallo-romaine à Lyon, II, 1980, no 387 : tête d'Apollon. Stéphanie Boucher, Bronzes romains du Musée des Beaux-Arts de Lyon, 1973, no 14 (Amour), 180 (Vénus), 199 et 200 (femmes), 203 et 204 (hommes). E. Espérandeu et H. Rol.IAND, Bronzes antiques de la Seine-Maritime, 1959, no 174 : enfant-Harpocrate? Ph. LAgrangE, Bronzes antiques découverts à Besançon, 1981, p. 77 (Apollon), p. 127 (aurige).

La hauteur de ces poids-curscurs varic cntre 5,5 et $11 \mathrm{~cm}$. Leur poids, rarement indiqué par les notices des catalogues, est le plus souvent de 500-700 g. Il peut atteindre plus exceptionnellement $1300 \mathrm{~g}$ el jusqu'à $11 \mathrm{~kg}$. Il s'agit bien d'objets d'un module tout different de celui du Mercure phallique du Mas-d'Agenais.

10 Voir par exemple Stéphanie Bơcher, Recherches sur les bronzes figurés de la Gaule pré-romaine el romaine, dans B.E.F.A.R. 228, Rome, 1976, pl. 47, nos 213-214; pl. 48, n० $222 ;$ pl. $55, n^{\circ} 264$. Les bustes accessoires des divinités panthées sont généralement soudés ou fondus sur l'armature qui leur sert de support (souvent des traverses posées sur l'extrémité d'une paire d'ailes).

11 Germaine Faider-Feytunns, op. cil., I, p. 159-162,
II, pl. 117, par exemple.

12 P. Cadevat, Nouvelles recherches..., p. 19-56. Présence de céramique campanienne $\mathbf{B}$ (p. 61) et de monnaies du II $^{\mathbf{e}}$ et du $\mathrm{r}^{\mathrm{e}}$ siècles (p. 264-265). Lne quinzaine de monnaies datent de l'époque pré-augustéenne mais "ont pu circuler jusqu'au principat de Claude (41-54) : "Quelques remarques " de D. Novy, p. 271 du même ouvrage.

13 Germaine Faider-Feytmans, Le Jupiter "de Bree" (Limbourg, Belgique), dans Bronzes hellénistiques et romains, tradition et renouveau, Actes du Ve colloque international sur les bronzes antiques, Lausanne, 1979, p. 183 et pl. 108, fig. 5. Id., Les bronzes romains de Belgique, I, p. 49-50 ; II, pl. 1 à $3, n^{\circ} 1$.

14 Stéphanie Boucher, Bronzes antiques de Vienne, Inventaire des Collections Publiques Françaises, no 17, Paris, K.M.N., 1971, p. 71-75, no 29.

15 F. Brammer, "Bronzes hellénistiques" et "romains". Problèmes de chronologie, dans Bronzes hellénistiques et romains... Actes du Ve colloque international sur les bronzes antiques, Lausanne, p. 37, pl. 13, fig. 17 el 18.

16 Germaine Faider-Feyrmans, Les bronzes romains de Belgique, I, p. 59-60; II, p. 14 et 15, no 29.

17 Les amulettes ne sont que très rarement datées dans les publications de mobilier ou les catalogues d'exposition. On ne peut donc se référer à une série pour préciser la datation du Mercure du Mas, a fortiori puisque l'on est en présence d'une pièce unique. Cf. par exemple, Germaine FAIDERFeytmans, op. cit., I, p. 159-162; II, pl. 117. 
2 Tête phallique à cirrus, de Bavai (Nord). (H. : $5,5 \mathrm{~cm}$ ) (d'après Germaine Faider-Feytmans. Recueil des bronzes de Bavai, pl. XLVI, no 292).

3 Tête phallique du Musée Aubanel, Avignon, prov. inc. (II. : $4,2 \mathrm{~cm}$ ). a : face; b : profil à gauche (d'après $\mathrm{H}$. Rolland, Bronzes antiques de Ilaute-Provence, no 196).
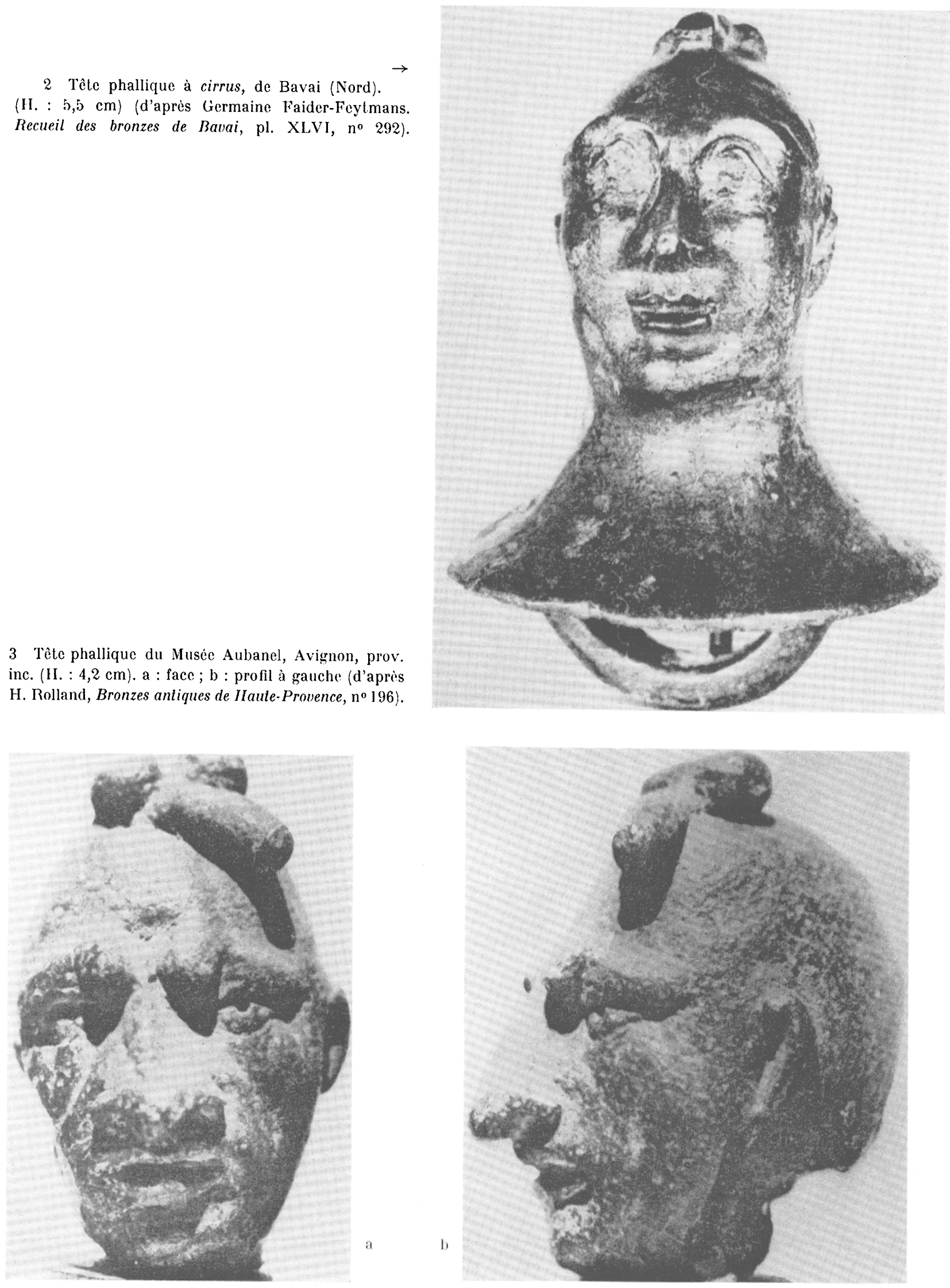


\section{Présence du phallus frontal}

Le caractère étonnant de l'amulette vient, naturellement, de l'appendice frontal qui en fait un objet unique. La présence incongrue d'un phallus érigé sur le front, en forte saillie, à la place de la touffe capillaire que l'on peut voir sur le Mercure de Saint-Marcel ou sur la statuette de Thiennes, fait de cette amulette une curiosité (fig. 1).

\section{II - ÉlÉments de COMPARAISON}

Il est évidemment possible de rapprocher cette amulette des "grotesques " ct autres pygmées désarticulés, ces nabots au phallus hypertrophié dont l'iconographie prend sa source dans le goût de l'exotisme, mais aussi au registre de la pantomime et des scènes de genre ${ }^{18}$. Mais ce serait considérer le Mercure du Mas-d'Agenais comme une "gauloiserie», une fantaisic grossière et irrévérencieuse de l'artisan bronzier.

En revanche, cette amulette prend un relief tout différent si l'on évoque les quelques documents dans lesquels le phallus se trouve curicusement associé à une tête, ou bien qui mêlent les figurations phalliques aux manifestations du culte rendu à Mercure.

\section{Les têtes phalliques au cirrus}

Les documents les plus proches par leur iconographie nous semblent être constitués par l'abondante série des prétendues " têtes d'Isiaques » dont certains rares exemplaires sont affublés d'un phallus renversé posé sur le crâne (fig. 2 et 3 ), le membre tourné vers le front ou, au contraire, érigé. Il s'agit des têtes au cirrus, appliques ou ornements de bronze d'un usage mal défini, le plus souvent destinés au décor des meubles (lits ou coffres de chambres à coucher, mobilier de lupanar?) ${ }^{19}$. Certaines d'entre elles avaient été ultérieurement remplies de plomb pour être remployées comme poids-curseurs de balances à fléau ${ }^{20}$.

18 Germaine FaIDER-F EYTMAns, op. cit., I, p. 128, no 224 ; II, pl. 91. P. LébeL, Stéphanie Bouchrr, Bronzes figurés antiques du Musée Rolin d'Autun, Autun, 1975, p. 71, no 114 ; Stéphanie Boucher, Recherches, p. 187-190, pl. 70, nos 337339.

19 J. Schwartz, Sur de prétendus isiaques, dans Latomus, XXII, 1963, p. 472-477, et pl. XLIX. I. RichaRd, Encore de prétendus Isiaques, dans Latomus, XXXII, 1973, p. 170-176. Stéphanie Boucine, Recherches..., p. 186-187, et p. 392-393, carte XXIV. J.-J. Hatt penche pour le décor du mobilier de chambres de lupanars.

20 On peut citer :

a. Tête phallique de Bavai, inv. no c. 394 du Musée de Mariemont (Belgiquc), fig. 2. Provenance incertaine ; attribuée à Bavai. Phallus renversé au sommet du crâne. Pièce d'applique, creuse à l'arrière, avec anneau de fixation à la base et en arrière, identifiè à un ornement de char. Germaine FAIDER-FEyTMANs, Recueil des bronzes de Bavai, VIII e suppl. à Gallia, 1957, p. 118, no 292, et pl. XLVI. Les Antiquilés du Musée de Mariemont, catalogue, 1952, p. 175, pl. 61, F. 27, no 394.

b. Tête phallique de Bavai, inv. no 605 du Musée de Douai (perdue, simplement connue par un dessin). Un détail en relief au sommet du crâne, jusqu'ici identifié comme le cirrus, nous paraît être plutôt le vestige d'un phallus renversé (vu de face et aplati, voire limé) que celui du cirrus, plus souvent placé sur l'occiput qu'au sommet du cråne. Germaine FAIDIR-FEXTMANS, Bavai, p. 118, n०293, et pl. XLVI.

c. Tête phallique de Bavai (aujourd'hui perdue), à "longue mèche de cheveux tressés, au centre " el "coifféc d'un phallus". Germaine Faider-FeYTMans, Bavai, p. 118 , no 294.

d. Tête phallique de Trêves, inv. no 5017 du Rheinisches Landesmuseum Trier. Phallus renversé sur le sommet du crâne d'une têle caricaturale au modelé grossier et aux rides accusées. II. Menzel, Die römischen Bronzen aus Deutschland, II, Trier, Mayence, 1966, p. 75 , no 180 , et pl. 61 .

e. Tête phallique de Trêves, inv. no G 73 du Rheinisches Landesmuseum Trier. Ornement de char trouvé dans la Moselle. Phallus renversé sur le sommel du cråne. H. MEnzer., Trier, p. $111, \mathrm{n}^{\circ} 270$, et pl. 82 .

f. Tête phallique de Strasbourg, inv. no 21.871 du Musée Archéologique de Strasbourg. Tête à phallus renversé sur le sommet du crâne, montée sur une soie et attribuée par J.-J. Hatt à l'ornement somital d'une hampe sacerdotale, fig. 3. J.-J. HATT, Observalions sur quelques slaluelles galloromaines en bronze du Musée de Strasbourg, Essai d'étude stylistique et de classement chronologique, dans R.A.E., XII, 1961 , p. 121-125, fig. 38 et 39 .

g. Tête phallique de provenance inconnue, collection Théodore Aubanel en Avignon. Phallus important, renversé sur la tête et déviant, sur le front ridé, vers la gauche du 


\section{Les dieux-faces phalliques de Saintonge}

Une seconde série de documents est constituéc par un ensemble de reliefs, celui des étranges dieuxfaces, phalliques ou non, de la Saintonge romaine (fig. 4 à 7 ).

Sur les reliefs calcaires grossièrement rectangulaires, parfois limités par un cadre, des visages masculins plus ou moins monstrueux sont sculptés de manière très fruste, grimaçants et inquétants : grands yeux exorbités à la fois fixes, inexpressifs et perçants, longues oreilles animales, pointues et dressées au-dessus des sourcils, arcades sourcilières puissantes soulignant un haut front chauve, tout plissé de rides, bouche hypertrophiée laissant pendre souvent une langue parfois multiple. Plusieurs de ces faces présentent des traits communs aux modillons à "masques feuillus" des corniches des grands monuments d'époque sévérienne ${ }^{21}$, ce qui incite à les dater de la fin du II $^{\mathrm{e}}$ siècle ou du $\mathrm{III}^{\mathrm{e}}$ siècle.

La plupart de ces masques présentent en outre des figurations phalliques indiscutables, notamment sur le front, tantôt pendantes, tantôt érigées, et souvent multiples ${ }^{22}$.

visage, fig. 3. A rapprocher des figurations de phallus obliques sur le front des dieux-faces du Musée archéologique de Saintes. II. Rotrann, Bronzes antiques de Haule-Provence, XVII1 suppl. à Gallia, 1965, p. 106, no 196 et fig. 196.

h. Tête phallique de provenance inconnue, inv. no A 4, 499 du Museo Archeologico de Vérone (Italie). Le phallus renversé posé sur la tête a êté supprimé ultérieurement par l'intervento drastico della lima. Attribué au $\mathrm{III}^{\mathrm{e}}$ siècle. L. Franzoni, Bronzelli Romani del Museo Archeologico di Verona, Venise, Alficri éd., 1973, p. 201, no 174.

i. Tête phallique de provenance inconnue, inv. $\Lambda 4,500 \mathrm{du}$ Museo Archeologico de Vérone (Italie). Phallus renversé, limé, sur le sommet du crâne. L. Franzoni, op. cit., p. 202, no 175 .

La présence à Vérone, c'est-à-dire en dehors de la zone "normale " de diffusion, le Nord et l'Est de la Gaule, de deux lêtes phalliques à cirrus ne laisse pas de surprendre. Ou bien c'est le butin de la collecte en Gaule d'un collectionneur averti el chanceux (deux exemplaires d'une série très rare), ou bien, ce qui est également possible, ces deux têtes phalliques à cirrus n'ont pas échoué là par hasard et bien que de " provenance inconnue "sont-elles d'origine locale ou régionale ? L'existence de deux exemplaires plaide en faveur de cette seconde hypothèse.

Notons qu'à Trêves et à Strasbourg comme à Bavai, il y a une "concentration" de têtes phalliques à cirrus qui correspond aussi, comme à Vérone, à une absence de tête à cirrus non phallique (sauf les deux bifrons non phalliques de Trêves et de Strasbourg). Il faut rappeler enfin que la région de Vérone fut marquée par l'expansion celtique.

j. Tête phallique de provenance inconnue du Metropolitan Museum of Art, New York (U.S.A.). Phallus renversé posé sur la tête, G. M. A. Richter, The Metropolitan Museum of Arl: Greek, Etruscan and Roman Bronzes, New York, 1915, no 276 .

k. Tête phallique, Musée Archéologique National de Naples. Phallus érigé dressé sur le front rasé d'un homme barbu et moustachu. J. Marcadé, L'Art el l'amour, Etrurie et Rome, Roma Amor, $2^{\mathrm{e}}$ éd., 1975, fig. p. 34. D. Mountfiel. , L'érotisme antique, trad. par B. Soulié, Vicence, Solar éd., 1982, p. 86.

21 L. Valensi, Bordeaux, 2000 ans d'histoire, cat. d'expo., Bordeaux, Musée d'Aquitaine, 1971, p. 43-44, nos 26 et 27.
22 Conférer :

a. Moitié supérieure d'un dieu-face phallique, inv. 49-485 du Musée archéologique de Saintes, fig. 5. Oreilles animales dressées, haut front chauve et ridé sur lequel pend un phallus, disposé en oblique vers l'oil gauche. L. Maurin, Saintes anlique, des origines à la fin du VI siècle après Jésus-Chrisl, Saintes, 1978 , p. 240 et p. 440 , fig. 311 .

b. Dieu-face polyphallique, inv. 82.19 du Musée archéologique de Saintes. Trois phallus (mutilés) pendent sur le front ridé, fig. 6 , le dieu tire une double langue, de part et d'autre du menton, dont la forme et la disposition évoquent l'iconographie des "masques feuillus" de la sculpture monumentale bordelaise attribuée à l'époque sévérienne (voir aussi, ci-dessous, d). Fin du II $^{\mathrm{e}}$ ou début du II $^{\mathrm{e}}$ siècle (?). L. Maurin, op. cit., p. 240, fig. 314 , et p. 441.

c. Dieu-face polyphallique, inv. 49.483 du Musée Archéologique de Saintes, fig. 7. Le dieu tire la langue. Deux phallus érigés s'opposent presque à l'horizontalc sur le front (l'un a presque totalement disparu). Phallus pendant le long de la joue droite et son symétrique le long de la joue gauche. Phallus érigé apparaissant au bas de la sculpture, à gauche, sous le menton. La partic droite du relief a été gravement mutilée mais les arrachements visibles et la symétrie des phallus du front laissent supposer une symétrie totale du relief et que le visage grimaçant était entouré d'une ronde de six phallus, double triphallie, tantôt érigés, tantôt pendants. Un septième phallus, érigé, était peut-être sculpté sur la face latérale gauche du relief. L. Maurin, op. cit., p. 240, fig. 312 ct p. 441. Le dicu ne porte pas d'anneau dans le nez. La trace circulaire sur la lévre supérieure est due à un phénomène d'altération de la pierre.

d. Un quatrième document phallique fait partie du décor du "Mausolée aux guirlandes " dont quelques blocs ornés sont conservés au Musée archéologique de Saintes (inv. 49-425). Il ne s'agit pas d'un relief isolé, mais d'un élément de la frise ornant l'intrados d'un cintre ornemental. A la suite de cinq masques feuillus, dont le schéma s'est développé, notamment en Bordelais, à la fin du $\mathrm{II}^{\mathrm{e}}$ siècle, un dernier visage dérivé des masques feuillus (traitement des joues et du menton), porte sur le front, juste au-dessus d'arcades sourcilières puissamment indiquées, un énorme phallus érigé, fig. 4. L. Maurin, op. cil., p. 129, 241 et 440, fig. 208. L. Maurin 


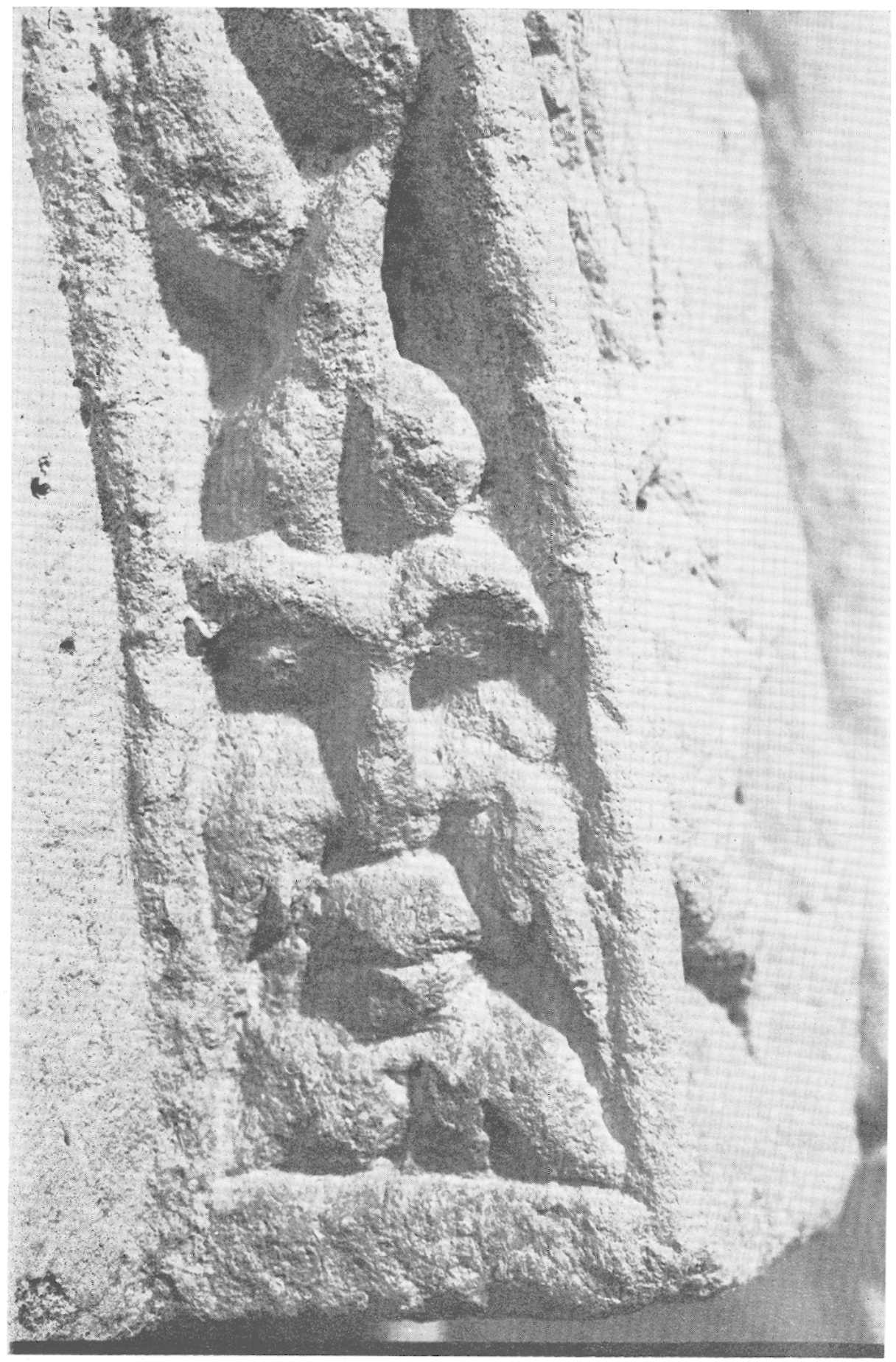

4 Dieu-face du Mausolée aux guirlandes de Saintes. (II. du masque : $15 \mathrm{~cm}$ ).

n'exclut pas que ce phallus ne soit qu'une simple feuille de lierre.

e. Enfin, peut-être faut-il rattacher à cette série l'une des cinq têtes colossales de Chorey (Côte-d'Or) : brisée en deux, elle présente une excroissance mutilée sur le front. Nous n'avons pu nous faire confirmer l'hypothèse d'une tête phallique, émise par L. Maurin, op. cit., p. 242, note 48. E. Thevenot, Sculptures inédites de Chorey, dans Gallia, V, 1947, p. 431, fig. 5; Id., A propos des têtes de Chorey (Côte-d'Or), dans Gallia, VI, 1948, I, p. 186-187. 


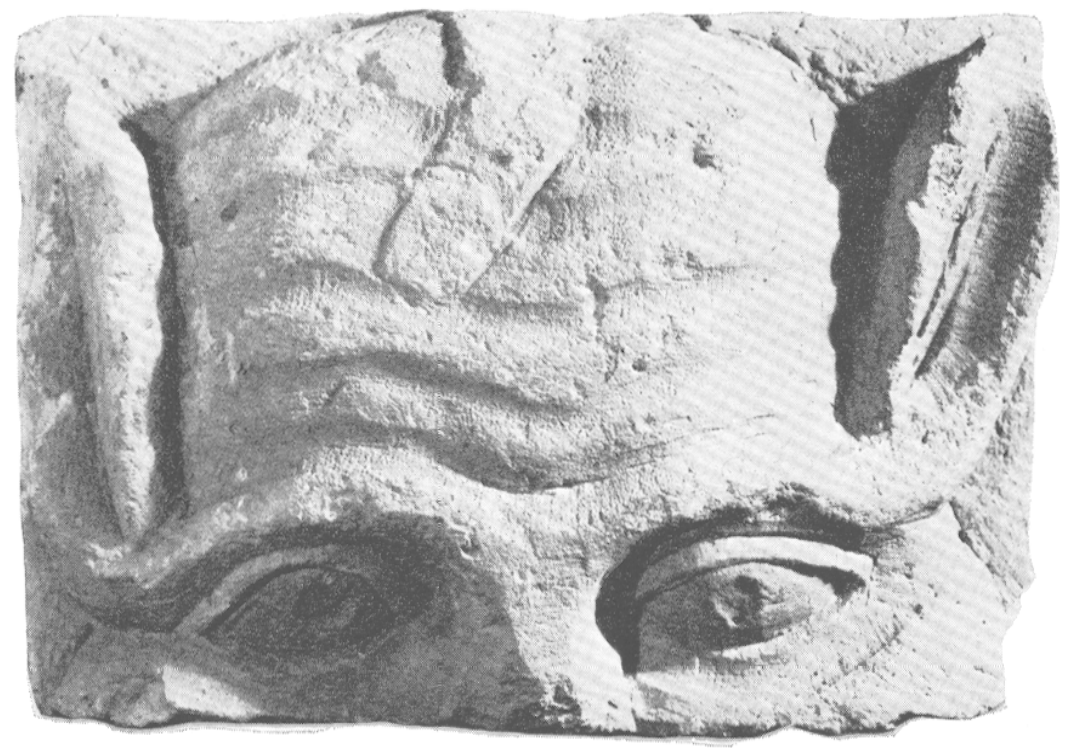

5 Dieu-face phallique de Saintes (Charente-Maritime) (inv. 49.485). (H. : $25 \mathrm{~cm}$ ).

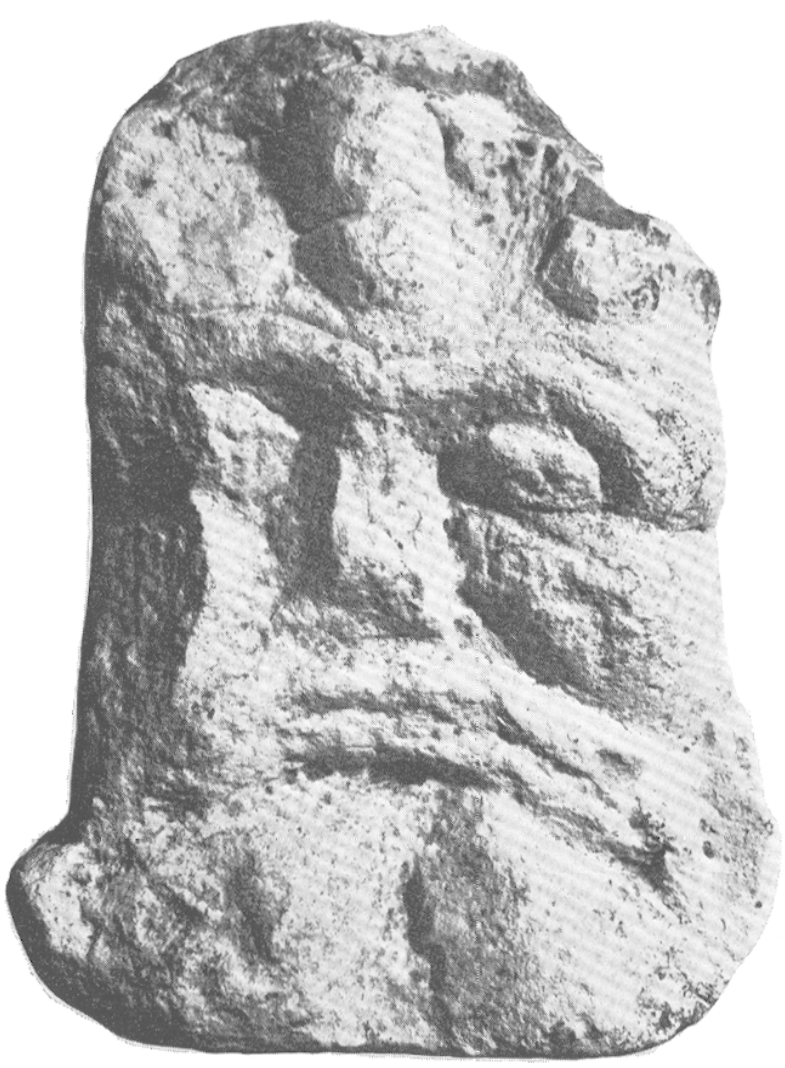

6 Dieu-face polyphallique de Saintes (inv. 82.19). (H. : $33 \mathrm{~cm}$ ).

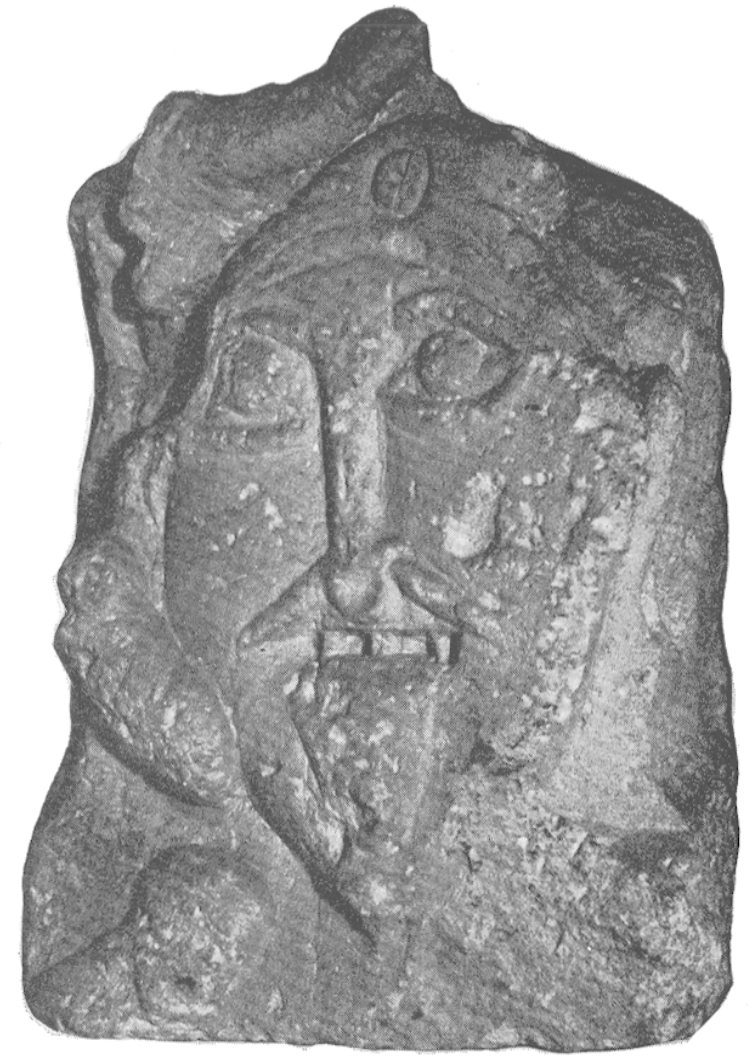

7 Dieu-face polyphallique de Saintes (inv. 49.483). (H.: $60 \mathrm{~cm}$ ).

\section{Le Mercure triphallique de Tongres}

Le Mercure triphallique de Tongres (Belgique), en bronze, est probablement le témoignage le plus évident de la relation étroite qui liait le phallus, une divinité gauloisc apparcntćc à Mercure et 
le Mercure des Romains ${ }^{23}$ : un pcrsonnage nu est assis, jambes écartées. Le membre viril a ćté mutilé. Il pouvait être hypertrophié. Le sujet tient une bourse de la main droite et un oiseau aux ailes déployées de la main gauche (coq? corbeau?). La tête est chauve et un phallus important, renversé, est posé sur le crâne dans l'axe du nez. Il a été perforé pour servir à la suspension de l'objet. Le nez est difforme, enflé, et pend comme un troisième phallus. La tête est chauve mais porte deux petites "loupes" de part et d'autre du phallus et une excroissance plus forte sur l'occiput (fig. 8).

\section{Mercure polyphallique de Naples}

Une statuette en bronze du Musée Archéologique National de Naples montre Mercure dans l'attitude de la course craintive (?), le corps penché en avant sur la jambe droite mais à demi-retourné vers sa gauche pour regarder par-dessus son épaule ${ }^{24}$ (fig. 9). De ses bras tendus en avant le dieu porte, comme un voleur son butin, le caducée (perdu) brandi de sa main gauche, et dans la droite, tenue par le col, la bourse allongée en forme d'outre. Les pieds sont nus mais les chevilles semblent porter des moignons d'ailerons. Une courte chlamyde plissée, nouée sur la poitrine, tombe sur les reins et laisse au dieu loule sa nudité. Mercure est coiffé du pétase ailé, ce chapeau aux bords ondulés en forme de carapace de tortue.

Outre son attitude inhabituclle, l'intérêt de cette statuctte pour le propos qui est le nôtre, vient de la présence de cinq phallus agressifs, dont la longueur exceptionnelle atteint celle des bras. Quatre d'entre eux naissent de la tête, sous le pétase, deux sur les tempes et deux sur l'occiput, et lui forment comme une étrange couronne radiéc. Au membre « principal » comme aux phallus adventices, les bourses sont inexistantes. Sous le gland du sexe divin et aux extrémités des deux phallus latéraux, une bélière servait, selon toute vraisemblance, à la suspension de trois clochettes dont les traces de chaincttes sont conservées çà et là.

A Naples encore, une peinture murale d'époque néronienne montre un personnage barbu et couronné de "fleurs", de lierre ou de vigne, courant à gauche. Un caducée dans la main gauche et des talonnières ailées le désignent comme le dieu Mercure. De sa main droite, il «tient, en laisse ", ou soutient à l'aide de bandelettes, un gigantesque phallus, érigé et luisant. On ne sait quelle beauté il poursuivait de son désir, mais la présence d'un pagne cachant son bassin, la suspension du monstrueux phallus (qui pourrait bien être une prothèsc) à l'aide de liens, la barbe ct la couronne fleurie (?) nous suggèrent qu'il s'agit ici d'un acteur plutôt que de la représentation du divin courrier. Il n'en reste pas moins qu'ici encore on a voulu montrer un Mercure phallique.

\section{Dieux ithyphalliques de Narbonne, d'Usseau et de Plougaslel.}

G. Picard évoque le moulage d'une statuette conservé sans origine au Musée des Antiquités nationales de Saint-Germain-en-Laye ${ }^{25}$. L'original, disparu, est cité par F. Benoit comme provenant de Narbonne. Il s'agit d'une statuette de Mercure "pourvue d'un énorme phallus redressé dont le dieu tient l'extrémité ».

23 Germaine Fainer-Frytmans, Les bronzes romains de Belgique, Mayence, 1979, I, p. 68 ; II, pl. 28. Inv. no 444 du Provinciaal (xallo-romeins Museum de Tongres (Limbourg, Belgique). Iraule de $8,8 \mathrm{~cm}$ et perforée pour suspension à son sommet, cette statuette a été trouvéc dans une sépulture. Elle est interprétée comme une amulette apotropaïque deslinće aux vivanls avant d'avoir été placée dans une tombe par référence au rôle psychopompe que les Anciens prêtaient à l'Hermès grec puis au Mercure des Romains.

24 Cetle statuette a été portée à notre connaissance par
F. Tassaux que nous remercions bien vivement. J. Marcadé, L'art et l'Amour. Etrurie el Rome, Roma Amor, $2^{\mathrm{e}}$ éd., 1975, fig. p. 64. Peinture murale du Musée Archéologique National de Naples : D. Mountrikin, L'érolisme antique, Vicence, Solar éd., 1982, p. 86 . Je remercic Mme R. Cantilena et M. O. de Cazanove qui m'ont aimablement communiqué la photographie du Mercure polyphallique de Naples.

25 G. Picarn, Dieux de fécondité dans la Gaule de l'Ouest, dans Acles du 10\%e Congrès National des Sociélés Savantes, Brest, 1982 , p. 125 et note 22. 

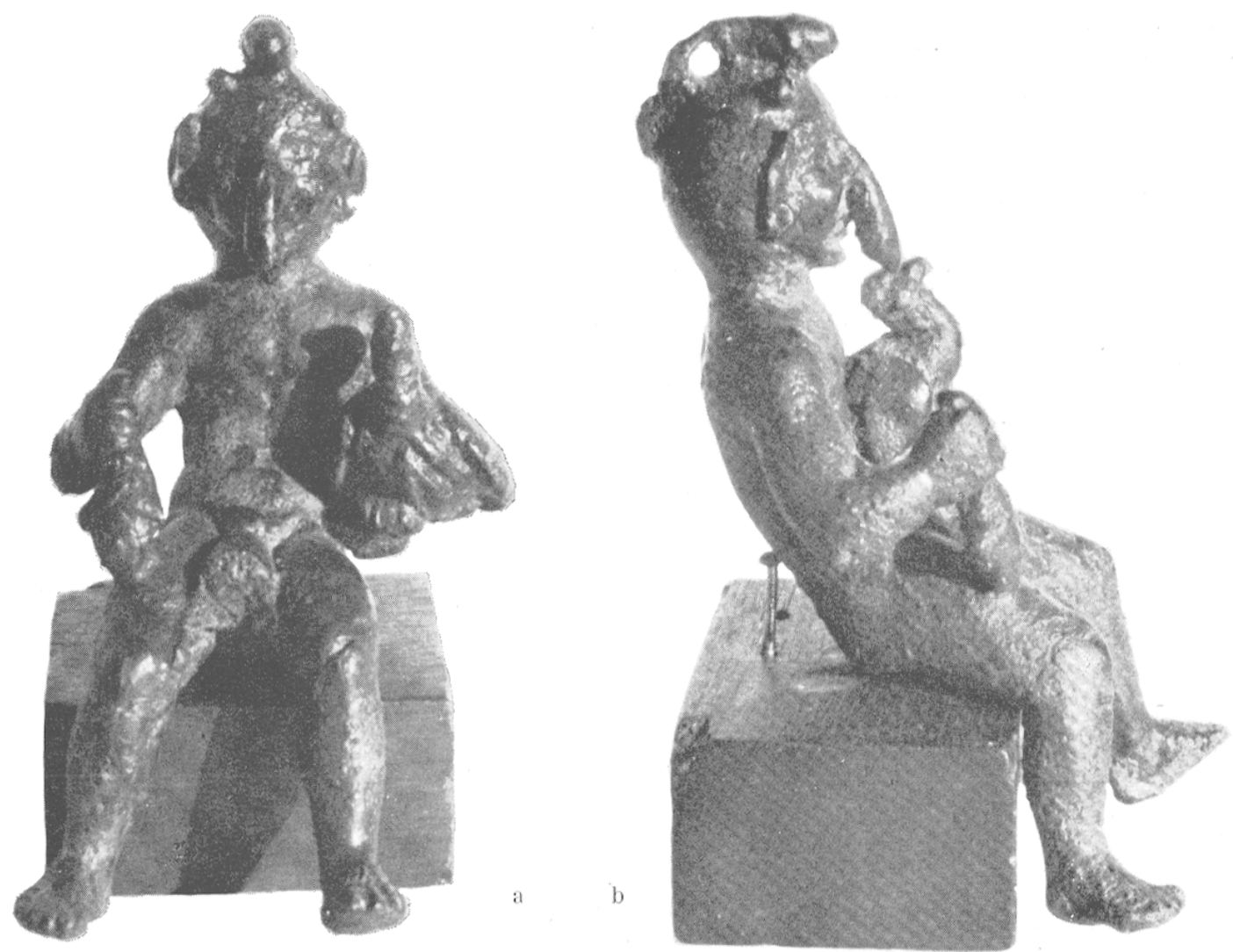

8 Mercure triphallique de Tongres (Belgique). (II. : $8,8 \mathrm{~cm}$ ). a : face; b : profil.

Cette même divinité phallique figure sur un relief inclus dans la base du calvaire de PlougastelDaoulas (Finistèrc) et sur une plaquette trouvée à Usseau (Deux-Sèvres) où il est associé à une déesse nue $^{26}$.

\section{Le graffite phallique de Poitiers}

Au faubourg de la Roche, à Poitiers (Vienne), dans le puits d'un temple dédié à Mercure, et notamment à la divinité gauloise vénérée sous le nom de Mercure Adsmerius, le P. de la Croix avait relevé plusieurs inscriptions et divers vestiges du culte rendu en ces licux. Sur les fragments de deux colonnes toscanes ramenées au jour, on peut lire, sur l'un, le graffite $M$ probablement pour Mercurio sacrum, sur l'autre, le graffite assez malhabile, sur trois lignes, IIIII / MIIR / CVRIOS, pour [...] / Mer/curios ou [...] / Mer/curio s(acrum), auquel était juxtaposé un phallus érigé, gravé horizontalement (fig. 10) ${ }^{27}$.

26 G. Picard, op. cil., p. 121 à 128.

27 C.I.L., XIII, 1126. R. P. C. de la Crolx, S.J., Les Temples et le puits de Mercure découverts sur les hauleurs de Poiliers en 1880, dans Mém. Soc. des Antiquaires de l'Ouest,

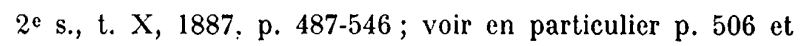
pl. 3, n$^{\circ} 5$. Sur le même sile, cruche de bronze consacrée à Mercure Adsmerius : C.I.L., XIII, 1125. 


\section{L'autel phallique à Mercure Augusle de Châlon-sur-Saône}

Dédié à Mercure Auguste, l'autel de Châlon-sur-Saône est une œuvre à la sculpture très fruste et à l'inscription malhabile, mais son iconographie est intéressante ${ }^{28}$ : le dieu est dcbout dans une niche, quasiment nu, la chlamyde attachée autour de son cou et flottant dans son dos. Les bras le long du corps, il tient une bourse dans sa main droite et un long caducée torsadé et tordu dans sa main gauche. A ses pieds figure la tortue. Les montants de la niche sont sculptés : le coq sur un socle ct le bouc, au-dessus duquel on voit un petit personnage habillé qui pourrait être le dédicant mais qui, pour J.-J. Hatt, serait plutôt le dieu gaulois Esus, associé à Mercure-Teutatès. Ce qui fait l'originalité de cet autel, c'est la présence, en relief, sur la face latérale droite, de deux phallus érigés, juxtaposés, obliques et représentés de profil.

\section{III - CoMmentaIRES ET hypothèses}

Ces différents documents ont des traits communs et s'éclairent mutuellement. Par unc chaîne d'analogies, ils nous conduisent à évoquer les survivances de la religion traditionnelle gauloise à l'époque romaine. Ces indications sont précieuses mais fragiles. Leur interprétation peut être contradictoire et nécessite la plus grande prudence. De même, la localisation de chaque trouvaille est importante. Cependant, si certains des petits bronzes phalliques découverts en Gaule s'avéraient d'origine italique, le fait même de les avoir rencontrés en Gaule montrerait qu'ils avaient trouvé un écho dans la mentalité gauloise.

\section{Réalité d'un "culle» phallique associé au culle d'un Mercure gaulois}

Le nombre même et la dispersion géographique des têtes phalliques, avec des regroupements notables à Bavai, Trêves, Strasbourg, Saintes et, peut-être, Vérone et Naples, montrent bien, quelle que soit l'interprétation que l'on pourra en donner, que l'adjonction du phallus sur une tête n'est ni fortuite, ni gratuite. Elle répond à une iconographie relativement précisc qui reflète un cliché, une superstition ou une croyance, et qui n'était pas liée à la seule fantaisie d'un bronzier.

La présence même d'un phallus érigé sur le front du Mercure du Mas-d'Agenais et la parure phallique des Mercures de Naples rendent caduc, s'il en était besoin, l'argument de l'interdit religieux en faveur de l'interprétation des têtes au cirrus comme têtes d'athlètes orientaux et non comme têtes d'Isiaques ${ }^{29}$. Non seulement le phallus du dieu du Mas ne saurait être sacrilège, surtout sur une pièce d'iconographie par ailleurs très "classique», mais il nous semble au contraire avoir été associé au culte du dicu ${ }^{30}$.

Cette idée d'un "culte " phallique ou, plutôt, d'un rôle religieux du phallus dans son association au culte de Mercure est niée par $\mathrm{B}$. Combet-Farnoux qui la juge «étrangère à la nature du Mercure

28 C.I.L., XIII, 2605. E. Espŕnannive, Recueil général des Bas-Reliefs, statues et bustes de la Gaule romaine, t. 3, 1910, p. 196-197, no 2132. Inscription Deo Mercurio Augu(sto) sacro / sacrum / Habro Aviti (filius). J.-J. HATr, Hermès dionysophore, image hellenisée d'un mythe gaulois, dans R.A.E., XVIII, 2, 1967, p. 4-9, fig. 3 .

29 Stéphanie Boucher, Recherches..., p. 187 : "la mèche, le cirrus, pourrait être empruntéc non aux représentations isiaques, dont le caractère religieux aurait interdit peut-être une telle désacralisation, mais bien plutôt à des figurations d'athlètes". Mais alors, que penser de la "triple désacralisation "du Vercure de Tongres, quintuple sur la statuette de Naples?

30 F. BlisorT, Mars et Mercure, nouvelles recherches sur l'interprétation gauloise des divinites romaines, dans Annales de la Fac. d'Aix-en-Provence, n. s. 25, 1959, p. 161. L'auteur considère que "le culte du phallus, principe de la vie, s'adresse à la force créatrice du Logos. Le phallus apparaîl alors comme l'un des allributs de Mercure en qui les Grecs voyaient le "Logos". 


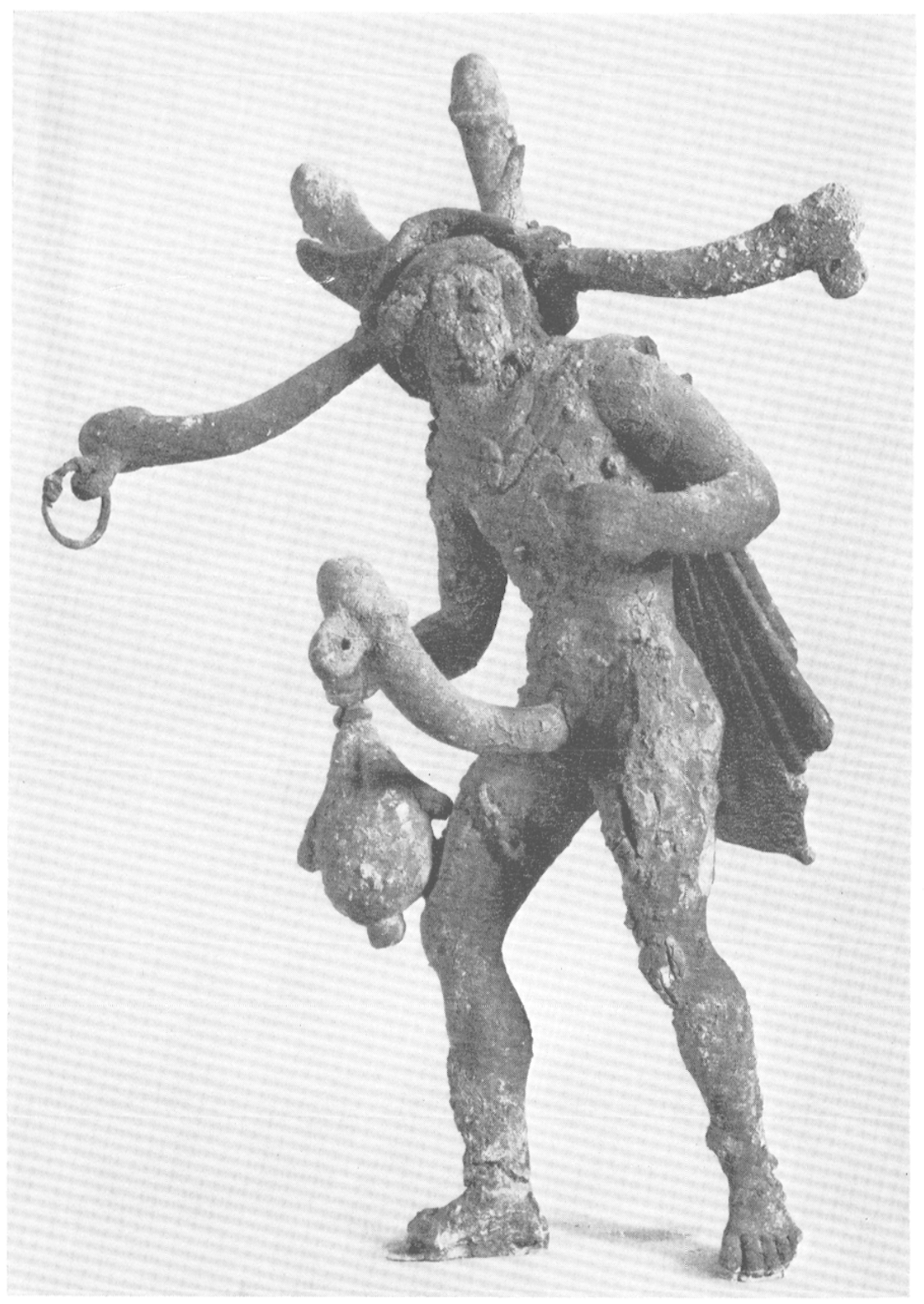

9 Mercure polyphallique de Naples (Italie), Musée National Archéologique. (H. : $14 \mathrm{~cm})$.

italique $»^{31}$, du moins à l'origine et jusqu'à l'époque augustéenne ${ }^{32}$. A. Legrand avait pourtant bien mis en lumière le rapport du Mercure originel, arcadien, avec la puissance sexuelle et le phallus ${ }^{33}$. Ce trait de la dévotion au Mercure gallo-romain est confirmé par la présence du phallus redoublé sur

31 В. Сомвет-Fannovx, Mercure romain, le culte public de Mercure et la fonction mercantile à Rome de la République archaïque d̀ l'époque augustéenne, dans B.E.F.A.R. 238, Rome, 1980 , p. 124 et 126.

32 La présence dans la Péninsule du Mercure polyphallique et de la fresque de Naples ne saurait, à elle seule, remettre fondamentalement en cause ce jugement. La relation entre Mercure et les figurations phalliques reste statistiquement marginale dans le domaine italique. Mais elle existe.

33 A. I.egraxd, s.v. Mercurius, dans Darembera et Saglio, Dictionnaire des Anliquilés, p. 1803 sq. "Nous savons qu'aux temps très anciens, il était figuré sous la forme significative d'un simple phallus " d'après les auteurs anciens, note 21 . 
l'autel, très fruste certes, mais d'iconographie classique, dédié à Mercure Auguste par Habro, fils d'Avitus, à Châlon-sur-Saône ${ }^{28}$. Cette allusion à une tradition gauloise, faite par un Gaulois, sur un autel dressé à la mode romaine, est tout à fait singulière. Elle est d'autant plus étonnante que le qualificatif d'Auguste associe manifestement le culte impérial au culte du Mercure romain qui recouvre ici, probablement, une divinité indigène, Esus et Teutatès tout à la fois, selon J.-J. Hatt.

Trouvé à Poitiers dans une enceinte sacrée et associé à des offrandes insignes comme ce vase d'airain précisément dédié à Mercurc Adsmerius, le sexe gravé à la suite du nom divin est lui-même l'évocation de la puissance créatrice, génératrice, du dieu invoqué27.

Le dieu triphallique de Tongres est lui aussi un témoignage très clair de l'association du Mercure des Romains, évoqué par ses attributs traditionnels (la bourse et le coq, ou le corbeau?), à une divinité indigène dont l'un des signes distinctifs serait le phallus, ici trois fois représenté23.

A l'inverse, le fait est étonnant que, parmi les nombreux poinçons sur céramique sigillée, domaine iconographique très sensible à la religion populaire, aucun de ceux qui ont été répertoriés par F. Oswald ne présente de caractère phallique particulier ${ }^{34}$.

En revanche, si l'on ne rencontre pas de têtes phalliques parmi les statuettes moulées en terre blanche du Centre de la France, les représentations ithyphalliques de Mercure n'y sont pas rares et le dieu a l'exclusivité de ce caractère. H. Vertet constate même chez les coroplathes arvernes "le souci de souligner le sexe » du dieu Mcrcure ${ }^{35}$.

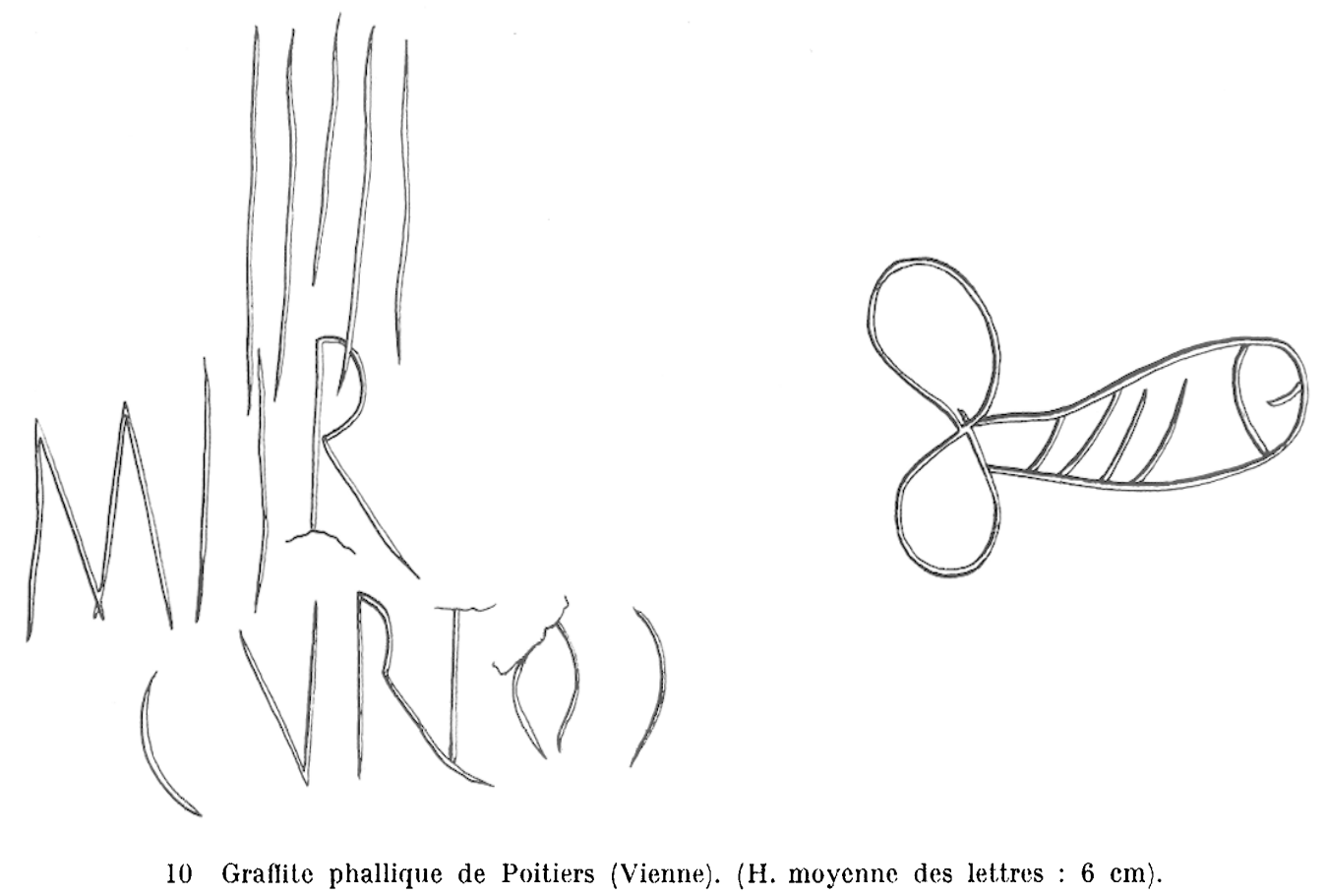

34 F. Oswald, Index of figure-lypes on terra sigillata, Liverpool, 1936-1937, nos 519 à 547.

35 H. Vertet, Remarques sur l'aspect et les altributs du Mercure gallo-romain populaire dans le Centre de la Gaule, extrait des Hommages à Albert Grenier, dans Latomus, LVIII, 1962, p. 1609-1610, pl. CCCXIX-CCCXXX, fig. 7-10. Ce même caractère est noté par E. THEvexot, Divinités et sanctuaires de la Graule, Paris, 1968, p. 84-85. 


\section{Le Mercure du Mas, le Mercure gaulois et les dieux-faces phalliques de Saintonge}

L'évidente association de la puissance phallique à l'image et au culte d'un Mercure gaulois qui a emprunté les attributs ou l'aspect du Mercure des Romains nous conduit, comme L. Maurin ${ }^{36}$, à voir dans les dieux-faces phalliques de Saintonge des représentations tardives d'une divinité locale qui, là, n'a pas eu besoin de se cacher sous les traits du Mercure de la religion officielle romaine. Se référant à ses oreilles animales qu'il attribue à un cervidé, J.-J. Hatt considère le dieu-face (fig. 5) comme une tête d'Esus, dieu du renouveau végétal et de la fécondité animale, dont l'une des fonctions aurait été de protéger la puissance sexuelle.

Il s'agit bien de figurations grossières par la médiocre qualité de la sculpture, et choquantes ou inquiétantes par leur caractère monstrueux. Mais là encore, la répétition des monuments et la redondance des attributs phalliques, dans une iconographie somme toute diversifiée, interdisent d'invoquer la fantaisie obscène pour expliquer ces reliefs. Penser différemment équivaudrait à nier tout fondement religieux au décor monumental roman dont le bestiaire fantastique et les nombreuses figurations impudiques n'étaient pas gratuits mais peuvent être souvent interprétées comme l'expression imagée, concrète, de concepts moraux, de préceptes religieux ou des conséquences tragiques et irréversibles de leur transgression par l'individu. Et c'est probablement le vague souvenir de cette iconographie oubliée que l'on retrouve dans le fabliau du $\mathrm{xIII}^{\mathrm{e}}$ ou du XIve siècle intitulé "Les quatre souhaits de Saint-Martin $»^{37}$.

\section{Les têtes phalliques au cirrus}

La disposition du phallus dans le type de la tête phallique au cirrus est manifestement fixée par une habitude iconographique : l'organe est toujours placé sur le sommet du crâne rasé; il est renversé, orienté vers le spectateur, mais il ne pend pas sur le front sauf dans le cas de la tête Aubanel (Avignon, cf. note 20, g, et fig. 3), de la tête de Naples (cf. note 20,k) et sur les dieux-faces de Saintonge (fig. 4 à 7). Le phallus est généralement placé dans l'axe du nez mais, sur la tête d'Avignon, il pend nettement vers l'arcade sourcilière gauche. Cette disposition est différente de celle du Mercure du Mas-d'Agenais ou de la tête de Naples. Le phallus y est en érection, le membre viril tourné vers le sommet du crâne.

Ces têtes phalliques au cirrus représentent un type oriental de personnage au crâne rasé à l'exception d'une mèche réservée sur l'occiput: le cirrus. A notre connaissance, ce type n'existe en Gaule que sous la forme de têtes sans corps même si, nous le verrons plus loin, deux statuettes de divinités assises peuvent lui être comparées. Avec le Mercure du Mas et les dieux-faces saintongeais, les onze têtes phalliques au cirrus que nous avons recensées confirment tout d'abord que l'association d'un

36 L. Maurin, op. cit., p. 241-242.

37 Anonyme du xir. ${ }^{\circ}$ ou du xive sic̀cle, Les qualre souhaits de Saint-Martin (M.R., V, CXXXIII, 201), dans Contes pour rire? Fabliaux des $X I I^{\mathrm{e}}$ et $X I V^{\mathrm{e}}$ siècles, Union Générale d'Éditions, Paris, coll. 10/18, no 1147, p. 58-63. Cie texte, aujourd'hui encore considéré comme obscène - il décrit les mésaventures d'un paysan et de sa femme qui obtiennent l'un et l'autre de voir pousser des sexes en chaque partie de leur corps - devait davantage faire rire au Moyen-Age, époque de grande liberté dans les mœurs, de la mésaventure et de l'inconséquence des protagonistes, de la faiblesse de l'Homme et de la mauvaise influence de la Femme, frivole, dans un ménage, que de l'évocation très libre et de la description des sexes : ... "Quand elle eut fini de souhaiter, les vits commencèrent à lui pousser. Ils lui saillirent dı nez, et par la bouche et de tous les côtés... Il n'y avait sur le vilain nul os ni membre dont ne saillit un vit merveilleux. Les vits sortirent même de ses genoux... Les vits lui sortent des oreilles. Par devant et puis en amont/Lui saillit un grand vit au front. / Et en aval jusques aux pieds / Le vilain fut de vits chargé. / De maint beau vit fut-il vêtu. / De toutes parts fut-il cornu..."

La morale de l'histoire : "Par ce fabliau vous pouvez voir / Que celui n'a guère savoir / Qui mieux croit sa femme que lui. / Il lui en vient honte et ennuis".

Ce texle nous a été aimablement indiqué par $R$. Bedon que nous remercions bien vivement. Peut-être a-l-il été inspiré par la survivance, ou la redécouverte, d'une statuette polyphallique analogue à celle de Naples? 
phallus à une tête isolée, à un dieu-lêle ${ }^{38}$ dans le sens évoqué par P. Lambrechts ${ }^{39}$, n'est pas chose fortuite.

Cette constatation nous conduit à nous interroger sur l'interprétation des "têtes d'Isiaques " ou "têtes d'athlètes orientaux au cirrus", phalliques ou non. En effet, malgré l'utilisation très diverse de ces objets dans la vie quotidienne (appliques ornementales, poids-curseurs, etc.) pour lesquelles les effigies divines du panthéon officiel furent, elles aussi, largement utilisées, il semble bien désormais que ces objets aient été porteurs d'une évocation religieuse ou à tout le moins superstitieuse : "plus que décorative et moins que religieuse au sens plein " comme l'écrit P. Veyne de l'imagerie bachique ${ }^{40}$. Cette manifestation phallique était censée être apotropaïque (elle devait chasser le mauvais œil) et propitiatoire (elle devait favoriser la puissance sexuelle) et de telles appliques se conçoivent fort bien (sans qu'on en ait la preuve) sur le mobilier de chambre à coucher voire de lupanar dans la Péninsule Italienne. Cependant, nous ne pouvons ignorer l'actuelle carte de répartition des têtes à cirrus qui montre la faveur relative dont ont bénéficié en Gaule, et notamment dans le Nord et dans l'Est, ces objets d'essence romaine, dont on sait aujourd'hui qu'ils ne sont pas totalement absents d'Italie (Vérone, Naples). Ils nous paraissent se rattacher peu ou prou aux symboles phalliques associés au culte d'un Mercure gaulois, qui pourrait être Esus ou une autre divinité polymorphe.

$\mathrm{Si}$, en raison des caractères physiques qu'il présente, l'origine « orientale » du type iconographique au cirrus reste probable, l'identification au portrait de prêtres d'Isis, habituels porteurs du cirrus, est désormais excluc. En effet, sur les onze têtes phalliques identifiées parmi les têtes à cirrus, sept sont d'origine connue et proviennent des régions du Nord ou de l'Est de la Gaule (Bavai, Trêves, Strasbourg) ou bien d'Italie (fig. 11). Cette distribution géographique confirme la faveur strictement occidentale de ces effigies dont la diffusion semblait limitée au Nord et à l'Est de la Gaule mais comprend également l'Italie ${ }^{41}$. Comme le rappelle Stéphanie Boucher, le type de la tête isolée au cirrus n'est pas connu en Égypte et la carte qu'elle en donne réfute de manière définitive l'hypothèse d'une origine "alexandrine " de ces objets ${ }^{42}$, hypothèse également écartée par la discussion de la composition chimique des bronzes dits "alexandrins" et sa comparaison à celle des bronzes "typiquement gaulois "43. De l'éventuelle relation de ce type de statuettes avec l'Égypte, il ne reste plus aujourd'hui que l'hypothèse bien improbablc d'un ćventucl prototypc nilotiquc dont on nc peut vérifier la réalité, faute d'en connaittre le moindre exemplaire. Au reste, plutôt qu'à l'image d'Égyptiens ou de Nubiens, ces têtes nous paraissent se référer à des types humains asiatiques (Europe Centrale ou Asie Mineure) ou, à tout le moins, levantins (sémites?).

En revanche, la découverte du Mercure du Mas-d'Agenais et la relecture du dieu triphallique de Tongres incitent à rapprocher ces fameuses têtes au cirrus du culte de Mercure. En effet, les deux petites « loupes " qui flanquent le phallus crânien du triphallique de Tongres pourraient être le souvenir des ailerons de tête du Mercure des Romains (ou de petites cornes?) et l'excroissance plus forte que l'on observe sur l'occiput rasé pourrait figurer une sorte de chignon aux lieu et place de l'habituel cirrus

38 Dans l'amulette du Mas-d'Agenais, le buste un peu disproportionné, et surtout coupé haut, semble avoir le rôle du socle d'un dieu-têle phallique, mais peut-être n'est-ce là que maladresse du sculpteur.

39 P. Lambrechrs, L'exaltation de la tête dans la pensée et dans l'art des Celtes, Dissertationes Archaeologicae Gandenses, II, De Tempel éd., Bruges, 1954.

40 P. Veyne, L'Empire romain, dans IIisloire de la vie privée, sous la direction de G. Duby, I, De l'Empire romain à l'an mil, p. 223 , note 3 .

Pour nous, la signification religieuse on superstitieuse du décor d'un objel n'exclut nullement une fonction décorative ni un rôle utilitaire. Ces aspect des choses est largement répandu dans le mobilier d'église ou même dans les breloques de nos porte-clefs (Saint-Christophe, chiffre 13 ou fer à cheval, par exemple).

41 Voir ci-dessus note $20, \mathrm{~h}$, i et $\mathrm{k}$.

42 Stéphanie Boucher, op. cit., p. 392-393, carte XXIV.

43 J. Condamin et Stéphanie Boucher, Recherches techniques sur des bronzes de Gaule romaine, IV, dans Gallia, 31,1973 , 1, p. 157-183, passim, et en particulier p. 158-160, 162, 165. Les bronzes dits "hellénisliques " ou "alexandrins", riches en ètain, en plomb et surtout en zinc, s'inscrivent dans une série gauloise de bronzes au zinc (plus de $8 \%$, parfois plus de $15 \%$ ). Les auteurs précisent qu'une proportion de $15 \%$ de zinc donne "une belle teinte or à l'alliage, ce qui pouvait ajouter à l'attrait de figurines exotiques, pilloresques ou grotesques". 


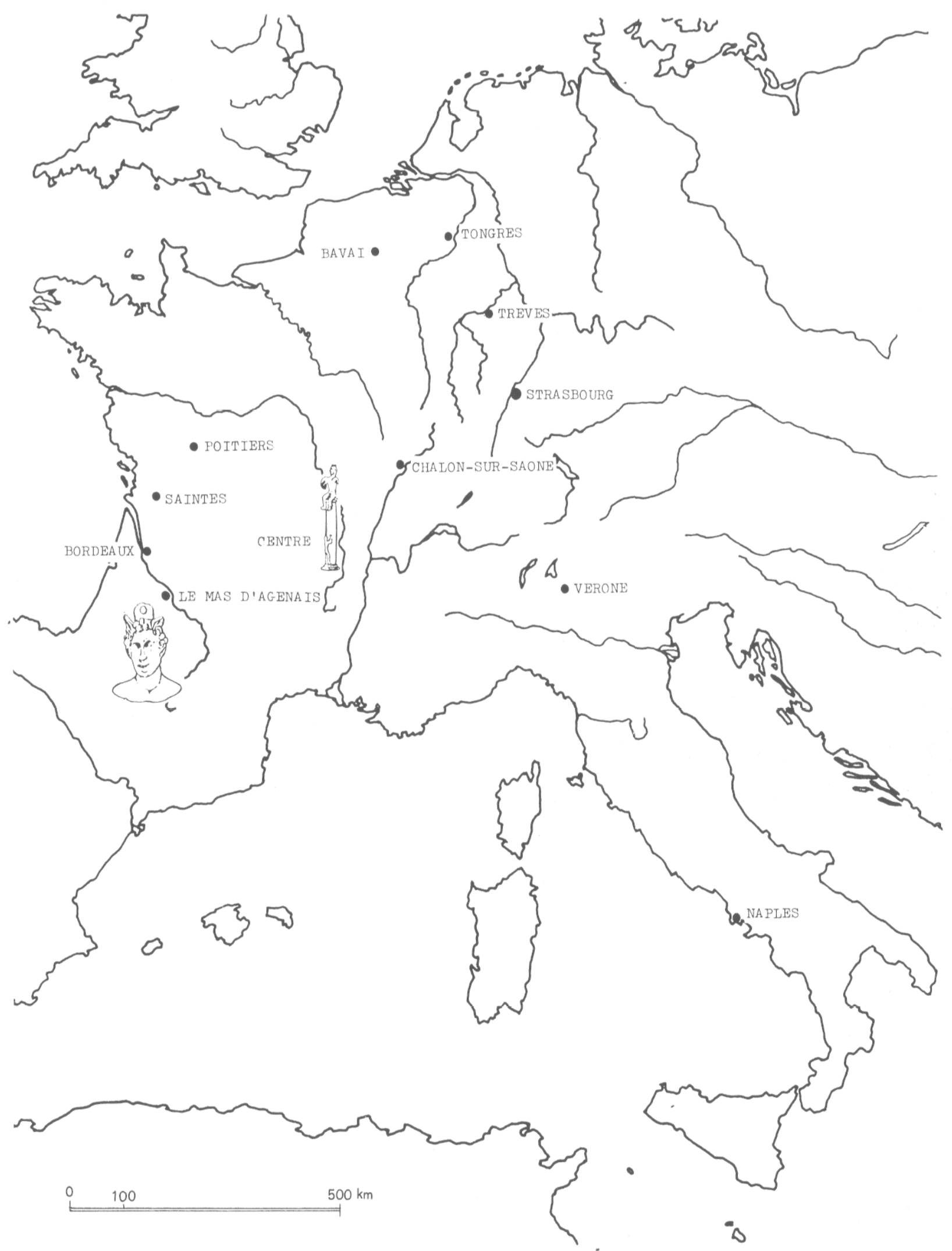

11 Carte des manifestations phalliques associées à Mercure. 
12 Amulette phallique à tête "orientalisante ", Trêves (Allemagne). (H. : 2,8 cm!

$\leftarrow$ (d'après H. Menzel, Trier, fig. 32).

13 Mercure quadricéphale de Bordeaux (Gironde). Cabinet des Médailles de la Bibliothèque Nationale. (H. : $9,1 \mathrm{~cm}$ ). a : profil à droite;

b : face ; c : profil à gauche (d'après un moulage).

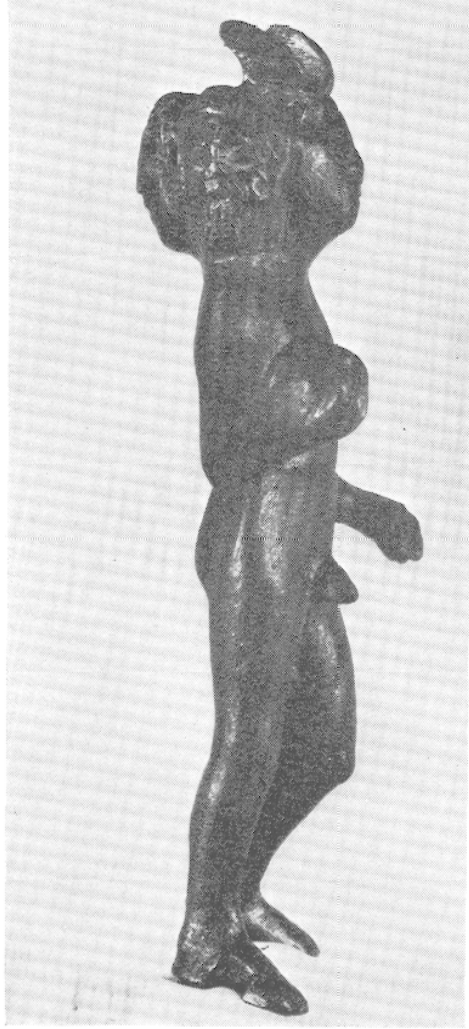

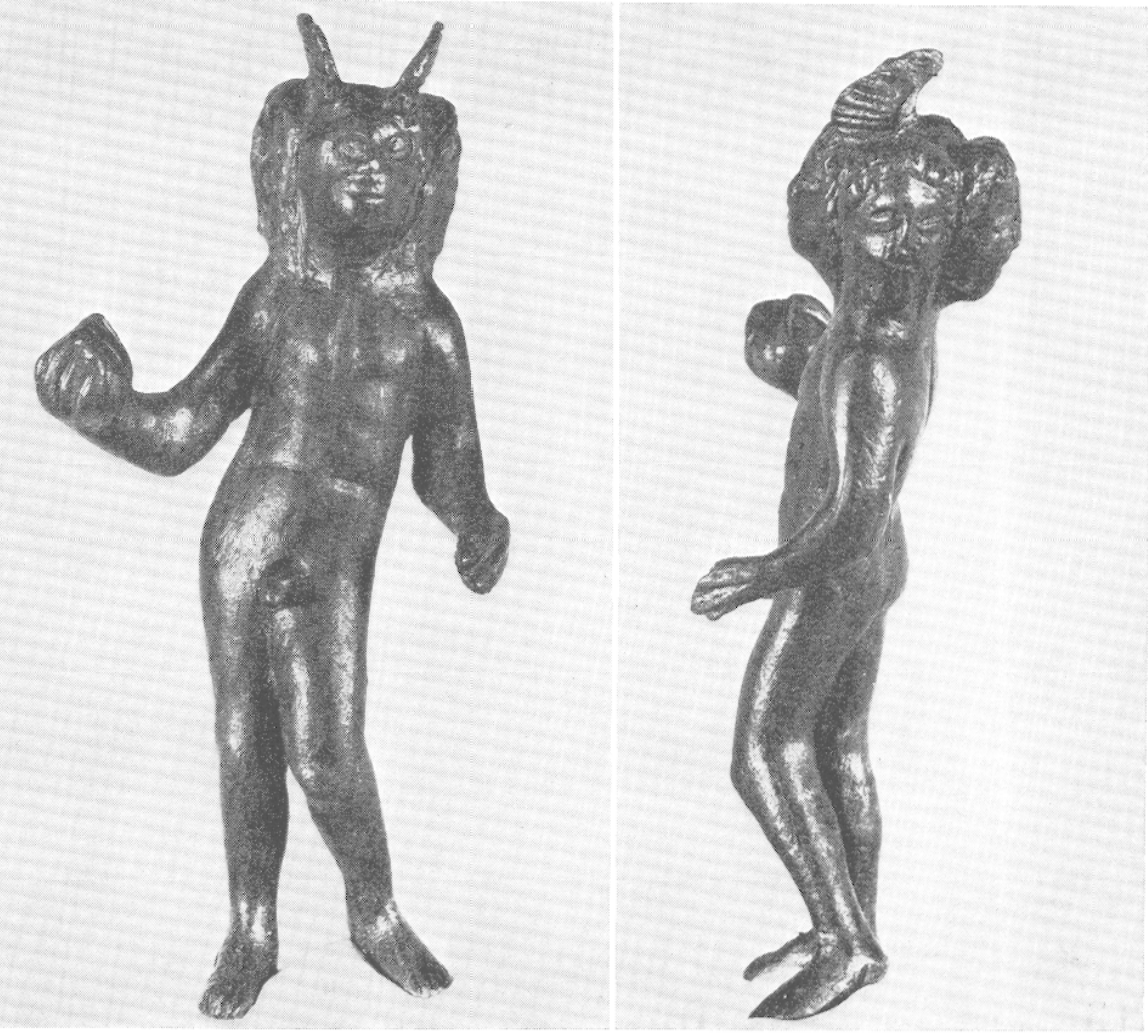

b

c

des têtes " orientalisantes 》 (fig. 8). Cette figuration divine complexe nous parait associer aux attributs du Mercure des Romains, la calvitie délibérée des pseudo-Isiaques au cirrus, la tracc probable de la mèche réservée et les caractères " ethniques 》 des têtes orientalisantes au cirrus (grands yeux exorbités, joues adipeuses, front fuyant, grandes oreilles écartées, nez busqué).

En faveur de l'hypothèse d'une parenté entre les têtes phalliques à cirrus et Mercure, nous constatons que les figurations phalliques non associées au culte de Priape se réfèrent exclusivement à un dieu proche de Mercure sinon à Mercure lui-même. Nous ne connaissons pas de Jupiter, d'Apollon, ni même de Mars, par exemple, qui soit porteur d'un tel attribut. En outre, P. Lambrechts observe, avec quelque étonnement, que, "de tous les dieux romains, seul Mercure semble avoir été conçu 
comme lête», dans le sens d'une effigie divine représentée en totalité par la figuration de son chef ${ }^{44}$. Il affirme aussi mais nous ne pouvons pas le suivre en cela, que les «dieux-têtes se caractérisent par l'absence totale de tout caractère phallique", ce que viennent désormais contredire les dieux-faces de Saintes.

Pour ces raisons, nous ne sommes pas loin de penser que les têtes phalliques au cirrus pourraient être assimilées, comme les dieux-faces de Saintonge, à la figuration d'une divinité indigène associée à Mercure. Cette hypothèse conduit à verser au dossier du Mercure gaulois, non seulement les têtes phalliques au cirrus, mais aussi, par extension, les pseudo-Isiaques non phalliques, plus nombreux ${ }^{45}$, et les rares têtes janiformes non phalliques, et nécessairement dépourvues de cirrus, qui présentent tous les caractères morphologiques des têtes dites orientalisantes. La carte XXIV de S. Boucher montre, en effet, la superposition de la diffusion des pseudo-Isiaques non phalliques et des têtes phalliques au cirrus. A Bavai, Trêves, Strasbourg, Naples, et, peut-être à Vérone, les bronziers ont jugé nécessaire de préciser encore les caractères du dieu représenté par l'adjonction du phallus. Peut-être étaient-ils mieux affirmés en ces endroits ou plus évidents ailleurs?

Un autre type d'association entre les têtes "orientalisantes 》 et le phallus est manifesté par deux amulettes de Trêves et de Blicquy (fig. 12) : un buste à tête "orientalisante " avec bélièrc sommitalc est prolongé par une plaque inférieure en forme de pelta, ornée d'un phallus pendant sur l'une, érigé sur l'autre ${ }^{46}$.

La distribution géographique des têtes phalliques coïncide également avec celle du phallusamulette observée par P. Lambrechts : "même à l'époque romaine, le phallus-amulette se rencontre surtout dans le nord-est de la Gaule " $^{47}$. Le même auteur note, comme B. Combet-Farnoux, "qu'en Italie, Mercure n'apparaît pour ainsi dire nulle part en relation avec le culte phallique ", mais nous avons vu les cas de Vérone et de Naples ${ }^{48}$. Si les Mercures de Naples n'impliquent pas un "culte", ils prouvent tout de même bien une association du phallus à l'image de Mercure.

\section{Les têtes "orientalisantes" janiformes, le Mercure gaulois et les hermès bicéphales celtiques}

L'hypothèse assimilant les têtes orientalisantes au cirrus, phalliques ou non, à une divinité indigène associée au Mercure des Romains nous conduit également à réviser l'opinion de P. Lambrechts sur les hermès doubles celtiques qui se distingueraient essentiellement de l'Hermès du monde grécoromain par "l'absence totale de caractère phallique, si nettement prononcé dans le cas de l'Hermès grec $\|^{49}$.

Ce caractère phallique des hermès bicéphales celtiques, à vrai dire tronqués ou mutilés aujourd'hui, pouvait être sous-jacent et les sculpteurs ont pu en faire l'économie comme dans le cas des têtes à cirrus non phalliques ou de certains dieux-faces saintongeais.

En effet, dans la série des têtes "orientalisantes" au cirrus, il existe un type bifrons, très rare. Certes les deux seuls exemplaires que nous ayons recensés, à Trêves (? ${ }^{50}$ et à Strasbourg ${ }^{51}$, sont

44 P. Lambreghts, op. cit., p. 71.

45 Stéphanie Boucher, Kecherches, p. 392-393 et carte XXIV.

46 H. MENzEL, Trier, p. 84-85, no 203, fig. 32. Inv. no 515 du Rheinisches Landesmuseum Trier. Cette amulette avait été considéréc comme une représentation de Priape. Elle est l'équivalent des têtes orientalisantes phalliques au cirrus. Même motif à Blicquy (Hainaut) : Germaine FAIDERFeytmans, Belgique, p. 161 et pl. 177 , no 314 .

47 P. LAmBrecirts, Note sur une statuette en bronze de Mercure au Musée de Tongres, dans l'Antiquilé classique, X, 1941 , p. 73.

48 Voir ci-dessus, note $20, \mathrm{n}^{\mathrm{os}} \mathrm{h}$, i et $\mathrm{k}$.

49 P. Lambrents, L'exaltation de la tête..., p. 71.

50 H. Menzel, Trier, p. 117 , no 285 et pl. 91 , de provenance inconnue, probablement locale ou régionale. Inv. n० G 66 du Rheinisches Landesmuseum Trier. Le buste bifrons jaillit d'un fleuron posé sur l'extrémité d'un doigt : fragment d'une main panthée? Cela confirmerait l'interprétation religieuse du sujet.

51 J.-J. Hatт, Sirasbourg, op. cit., p. 127-128, fig. 41 et 42. Trouvé à Strasbourg. Inv. no 7247 du Musée archéologique de Strasbourg. La présence, à la base du cou, de trois trous * dont un porte encore un anneau de chaînette ", suggère à l'auteur une utilisation comme sommet de hampe d'insigne sacerdotal (?). Il propose également d'interpréter les perforations de la base du cou comme les points d'attache de trois clochettes. On en voit souvent, en effet, à la base des bustes de divinités panthées. Selon Daremberg, Saglio et Pottier, op. cit., s.v. tintinnabula, "ces clochettes, tout comme le phallus, servaient à conjurer les mauvais esprits ". 
dépourvus de phallus et même de cirrus puisque les deux têtes sont accolées par l'occiput. Cependant, les caractères morphoscopiques de ces objets permettent de les classer dans la série des têtes "orientalisantes " à cirrus dont les relations avec un Mercure gaulois qui a pour attribut principal le phallus ont été montrées plus haut. Ces deux figurines bicéphales, "orientalisantes ", au crânc rasé, évoquent naturellement les hermès grecs. Mais elles doivent également être rapprochées du type celtique du dieu à deux faces accolées comme l'Hermès bicéphale de Roquepertuse (Bouches-du-Rhône) ${ }^{52}$, dont on peut supposer, d'après un passage de Grégoire de Tours, qu'il était souvent juché au sommet d'une colonne ${ }^{53}$, comme le Jupiter cavalier à l'anguipède.

Les deux têtes du bifrons "orientalisant" de Strasbourg ne sont pas semblables. L'une montre un visage plus âgé que l'autre même si les deux sont imberbes ${ }^{54}$. Ce trait évoque les bicéphales ou les tricéphales celtiques, ou dans la tradition celtique, dans lesquels alternent parfois visage glabre et tête barbue ${ }^{55}$. C'est aussi le cas du petit Mercure quadricéphale en bronze trouvé à Bordeaux qui montre, sous une unique paire d'ailerons, deux faces barbues associées à deux visages imberbes ${ }^{56}$ (fig. 13).

Naturellement ces bicéphales de bronze sont beaucoup plus tardifs (quatre ou cinq siècles) que les

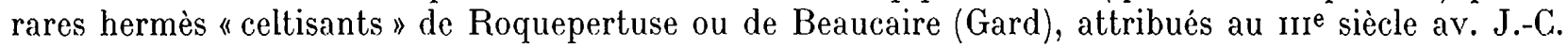
Toutefois, il faut observer que ces sculptures de pierre proviennent des marches du monde celtique, de cc littoral ligure non celtisé qui tint du contact avec le monde grec et avec les Ibères, la connaissance de l'architecture et de la sculpture en pierre quand le reste de la Gaulc ne travaillait encore que le bois. Là fut traduite dans le calcaire la statuaire en bois aujourd'hui disparue de la Gaule celtique. De la même manière, on peut considérer les bronzes bicéphales et les têtes à cirrus d'époque romaine comme la traduction tardive dans le bronze d'une statuaire gauloise en bois qui nous est inconnue.

Enfin, l'existence même des têtes "orientalisantes" janiformes étaye, à nos yeux, l'hypothèse selon laquelle les gallo-romains attribuaient bien à ces objets du décor quotidien une signification plus ou moins sacrée : ils y voyaient «la figuration, par la tête seule », fût-clle double ou triple, "d'un concept divin ».

\section{Trois têtes, trois sexes, trois cornes}

On a longtemps disserté sur l'étrangeté et la signification des divinités tricéphales du monde gaulois. Selon W. Deonna, "la répétition multiplie la puissance de celui ou de ce qui en est l'objet, mais sculc la répétition triple hausse cette puissance à son degré suprême, on pourrait dire inconcevable " ${ }^{57}$. Représenter une divinité avec trois têtes, c'est à la fois concentrer dans la tête toute l'essence divine mais aussi multiplier à l'infini la puissance du dieu.

52 Espérandieu 7616. I'. BenoIt, L'art primilif médilerranéen de la Vallee du Rhône, 1955, pl. XXX1V-XXXV. Les têtes paraissenl chauves mais, en réalité, la chevelure était figurć, par des traits rectilignes, juxtaposés, de peinture noire, en particulier au-dessus des tempes. Cette peinture a aujourd'hui disparu.

53 Grígorre de Tolrs, De passione et virlulibus sancli Iuliani marlyris, dans J. Swincikr, Fontes Historiae Religionis Celticae, II, p. 177, cité par P. LAmbrechrs, Note sur un passage de Grégoire de Tours relatif à la religion gauloise, dans Latomus, XIII, 1954, p. 207-217, et, en particulier, p. 213.

54 J.-J. IIATt, dans son Essai sur l'évolution de la religion gauloise, dans IR.E.A., LXVII, 1965, p. 105 et note 1, évoque "ce Janus bifrons, à facc guerrière et pacifique ".

55 I.es neuf divinités tricéphales trouvées à Reims, qui portent les attributs de Mercure, sont loutes à trois faces barbues (Espérandieu 3651 à 3661), de mème que le Lricéphale de Paris (Esp. 3137) ou le Cernumnos tricephale de Condat-sur-Trincou (Dordogne, Esp. 1316). Fin revanche, sur la base à quatre divinités de Reims (Esp. 3666), une divinité masculine vêtue, portant patère el baton noueux, présente un double visage, d'un côté jeune et imberbe, de l'autre barbu et âgé. Cette divinité bicéphale de Reims était distincte, aux yeux des gallo-romains, du Mercure des Romains, figuré selon une iconographie toule conventionnelle dans une autre niche du même monument.

56 Coll. du Cabinet des Médailles de la Bibliothèque Nationale, Stéphanie Bocchrr, Recherches..., p. 121, pl. 45, no 205 .

57 W. DEoxNa, Trois, superlatif absolu, da propos di taureau tricornu el du Mercure triphallique, dans l'Antiquité classique, XXIII, 1954, p. 419. 
Selon ce principe de la "répétition d'intensité " constaté dans l'iconographie celtique ${ }^{58}$, la triphallie (ou la polyphallie) aurait la même signification : les phallus multiples des dieux-faces de Saintes ou du Mercure de Tongres attirent l'attention sur l'organe, sur sa fonction (fécondité, puissance, abondance, prospérité, protection contre les maléfices) et ils en manifestent la surhumaine puissance. J.-J. Hatt au contraire voit dans le Mercure polyphallique de Naples la caricature du rôle propitiatoire du phallus. Pour lui, l'impressionnante parure phallique du dieu signifierait : "tu auras beau multiplier les phallus, tu resteras impuissant " et cela justifierait l'attitude de fuite craintive ou honteuse que l'on peut attribuer au Mercure de la statuette de Naples. Comme on le voit, si la relation Mercurephallus est maintenant établie, son interprétation est bien incertaine.

Considérant l'équivalence corne-phallus dans la représentation traditionnelle de la virilité5 ${ }^{5}$, W. Deonna constate la présence fréquente du bucrane ou de la tête de taureau dans les amulettes phalliques. Il cite en particulier une amulette du Cabinet des Médailles de la Bibliothèque Nationale qui montre trois phallus jaillissant de la gueule d'un bucrane ${ }^{60}$. Une amulette similaire a été trouvée dans le Hainaut ${ }^{61}$. Cette association manifeste encore que le taureau était un animal divin (on le sait associé aux trois grues sur la face inscrite TARVOS TRIGARANVS du pilier des Nautes de Paris), un symbole de la force régénératrice. Mais aussi, "la corne équivalant au phallus " ${ }^{62}$, le taureau tricornu $^{63}$ et le sanglier tricornu ${ }^{64}$, plus rare, pourraient être également associés à la divinité phallique, et même triphallique, dissimulée sous les traits de Mercure.

\section{Le Mercure triphallique de Tongres el Priape lriphallus}

A la calvitie délibérée, au souvenir (chignon?) de la mèche réservée occipitale et aux traits "orientalisants" des pseudo-Isiaques au cirrus, le Mercure triphallique de Tongres, figuration divine complexe, joint la bourse et le coq (?) du Mercure des Romains aux figurations phalliques redondantes des dieux-faces saintongeais. Pour W. Deonna, "Priape, qui n'est autre chose qu'un phallus anthropomorphisé $»^{65}$, était lui aussi, réputé "triphallus ${ }^{66}$. Cependant, on ne peut soupçonner Priape de contamination avec Mercure et, en l'état de nos connaissances, il n'existe pas de figuration de Priape triphallique. Les documents que nous avons rassemblés montrent assez qu'en Gaule, mais en Italie également, les figurations phalliques ne sont pas l'apanage de Priape (qui, au demeurant, fut assez peu honoré en Gaule), ni de Bacchus ou de son entourage. Au contraire, dans tous ces cas, le «culte " phallique est bien associé aux images d'un Mercure gaulois, voire à la représentation marginale d'un Mercure italique.

58 Le souvenir de la tricéphalie celtique se manifeste dans l'iconographie chrétienne dès l'époque romane pour les images de la Trinité. La représentation de la Trinité par un personnage tricéphale ou à triple visage, analogue aux tricéphales de Paris ou de Reims, a été condamnée comme, hérétique par le Concile de Trente (1515-1563). Cependant, une vignette ornant un livre d'heures de Rouen, datant probablement de 1588 , montre encore à celtc date une Trinité à triple visage (Musée Dobréc, Nantes, Cat. 1903, p. 23, $n^{\circ} 33$ ) qui paraît également sur un curieux relief du Xvie s. de l'église de Varangeville-sur-Mer (Seine-Maritime) et sur une peinture anonyme, du $\mathrm{xIx}^{\mathrm{e}} \mathrm{s}$. probablement, conservée au Musée Dobrée (inv. 958.2.12). Cf. I.. Rriau, L'iconographie de l'art chrétien, II, 1, 1956, p. 14-15 et 21-22. Communication de M. Berthail, Musée Dobríe, et de Ph. Sorel, Musée Carnavalet, Paris.

59 Cette équivalence existe aujourd'hui encore en Italie où lon vend force breloques ou porte-clefs formés d'une corne rouge couronnéc.
60 W. Deoriva, op. cil., p. 428. E. BABELON et J.-A. Bla.CuEr, Catalogue des bronzes antiques de la Bibliothèque Nationale, Cabinet des Médailles, Paris, 1985, p. 482, no 1175.

61 Germaine Faider-Feytmans, Belgique..., p. 161 et pl. $117, \mathrm{n}^{\circ} 315$.

62 Cette équivalence cst manifestéc cncorc au Moyen- $\Lambda$ gc par ces vers des Quatre Souhaits de Saint-Martin : ... "que vous soyez de vits chargé / ... Que toujours soient les vits tendus, / Que vous sembliez l'homme cornu ». Voir plus haut, note 37 .

63 Stéphanie Boucuse, Recherches..., p. 170-173, pl. 65, nos 310 à 313 par exemple.

64 Stéphanie Boucher, Recherches..., p. 173, pl. 66, $\mathrm{n}^{\circ} 316$.

65 W. DeonNa, De Télesphore au "moine bourru", dieux, génies el démons encapuchonnés, dans Latomus, XXI, 1955, p. 79 .

66 W. DronNa, Trois, superlatif absolu..., p. 424. 


\section{Origine du Mercure phallique}

G. Picard dit du Mercure de Tongres qu'il s'agit d'une "divinité qui n'est pas d'origine celtique, quoiqu'on répète, mais qui répond à un type grec primitif, romanisé tardivement ${ }^{67}$ et W. Deonna, après lui, estime que l'origine de cette statuette "ne doit pas être cherchée en Gaule mais au Sud "68. La statuette et la fresque de Naples semblent leur donner raison et prouvent en tout cas l'existence d'un Mercure phallique italique, fût-il caricatural.

Pour la formation du motif iconographique du phallus hypertrophié considéré comme un organe autonome ou une personne (cf. les amulettes phalliques), il faut probablement se référcr au monde méditerranéen le plus ancien même si la carte de répartition des amulettes phalliques montre actuellement une zone de plus grande fréquence dans le Nord et l'Est de la Gaule. Cependant, nous ne savons pas si nous devons ce développement des représentations phalliques sur le territoire de la Gaule à un concept religieux autochtone ou, au contraire, à l'influence d'une croyance méditerranéenne.

\section{IV - UN DIEU STYLITE INÉdit}

La curieuse statuette de Tongres montre une divinité assise mais la position de ses jambes diffère de celle que l'on observe généralement chez un personnage assis sur un siège : jambes presque jointives, cuisses à l'horizontale formant un angle à peu près droit avec le torse, ou au contraire jambes écartées et décalées, et torse oblique, penché en arrière, comme dans le cas des Mercures assis sur un rocher, du type du Mercure de Feurs. Ici, les jambes sont écartées et pendantes. Si l'on redresse la statuette jusqu'à ce que le buste en soit vertical et que ses avant-bras, qui présentent les attributs, soient horizontaux, on se rend compte que la position des jambes devient "anormale ", que les cuisses sont obliques et pendantes, que les talons sont légèrement ramenés sous le sujet : c'est l'attitude d'un personnage chevauchant un animal ou, comme on va le voir, assis jambes pendantes au sommet d'une colonne.

Cette observation nous conduit à faire un rapprochement avec une statuette inédite en bronze, tout aussi étrange ${ }^{69}$ (fig. 14) : sur une colonne toscane au renflement bien marqué, à section ovale (maladresse), à large base pseudo-circulaire ornée d'un double tore, est assis, sur un "chapiteau " à deux tores, un personnage totalement nu dont les bras ont été remplacés par deux ailes à demidéployées. L'aile gauche est brisée. Le visage est ovale, quelque peu bouffi.

Légèrement retroussé, le menton est bifide et imberbe. Une lèvre supérieure débordante, un nez busqué et des yeux assombris par des arcades sourcilières puissamment indiquées, donnent un aspect sévère au personnage. A peine marquées, les larges oreilles ont été quelque peu effacées par l'ébarbage et les retouches sommaires dues à l'artisan. Le crâne rasé ne présente aucune indication de chevelure. En revanche, deux excroissances à son sommet pourraient être de petits ailerons ou, plutôt, de petites cornes $^{70}$.

Les détails anatomiques sont traités de façon sommaire : des incisions figurent les rémiges sur la face antéricure de l'aile droite. Les formes sont grossièrement modelées et les masses musculaires sont souvent asymétriques. Le sexe du dieu semble absent.

67 G. PICARD, R.E.L., XXXI, 1953 (1954), p. 392.

68 W. Deonia, op. cit., p. 427.

69 Ancienne colleclion de M. Paul Roudié, Professeur honoraire à l'Université de Bordeaux III, qui en a fait le don au Musée d'Aquitaine à Bordeaux. Nous remercions vivement M. Roudié de nous avoir communiqué cet objet ennigmatique, d'avoir bien voulu nous autoriser à en proposer une première interprétation.
70 Ces excroissances sont aplaties dans le sens de l'épaisseur du sujet et font penser à des moignons d'ailerons mais cette épaisscur pourrait n'être due, comme l'ovale de la section de la colonne, qu'à une maladresse du modelage ou du moulage. Ce seraient donc plutôt des cornes grossic̀rement cylindriques, et non effilées, que l'artisan aurait voulu réaliser. Ces appendices ne semblent pas accidentellement tronqués. 


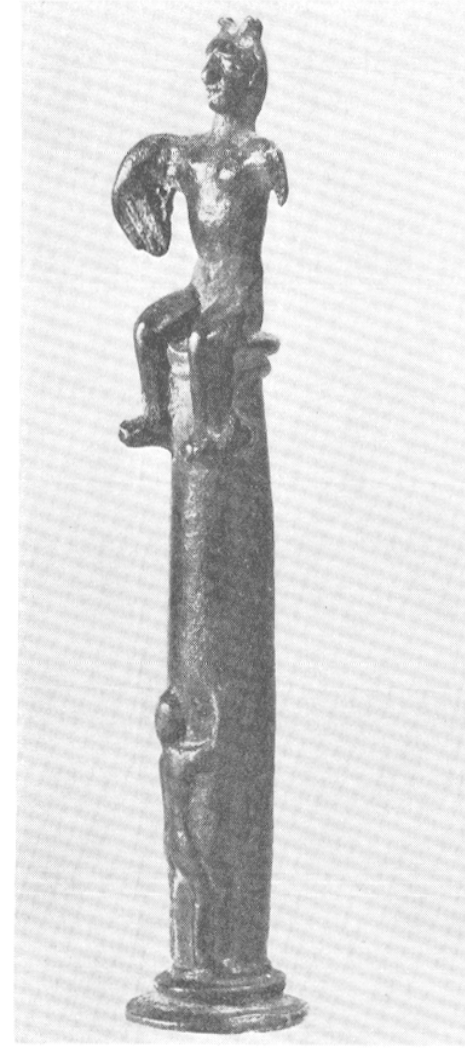

a

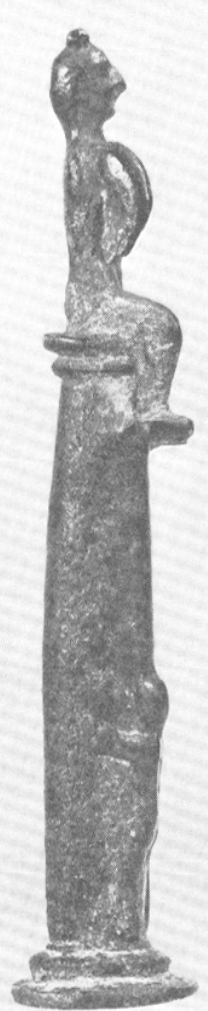

b)

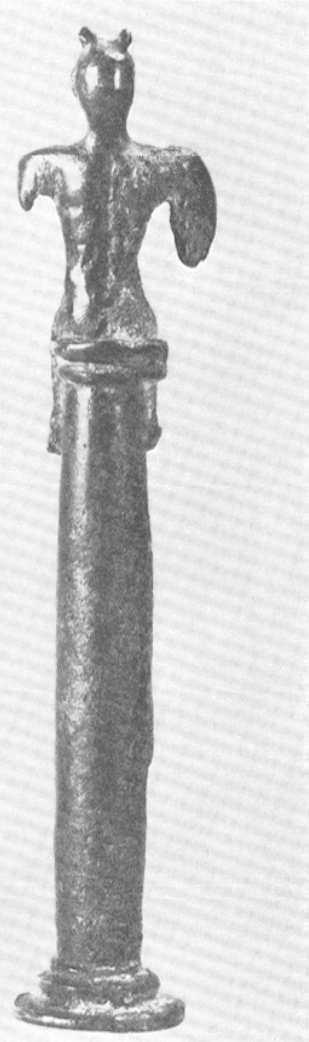

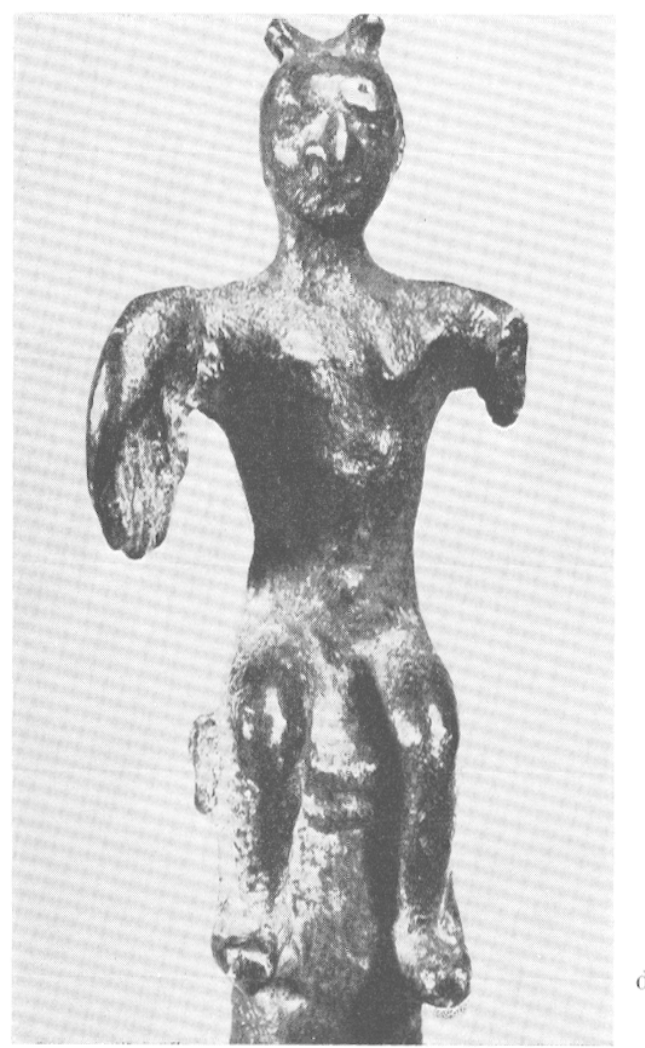

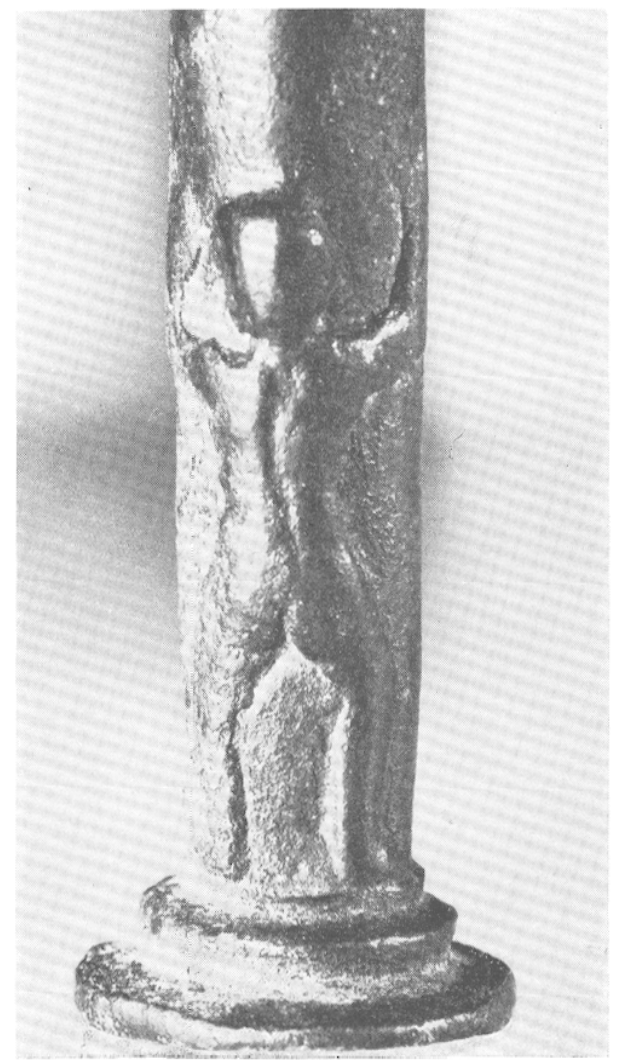

14 Dieu stylite (Centre de la France ?). (II. : 9,3 $\mathrm{cm}$ ). a : trois-quarts à gauche; b : profil à droite ; $\mathrm{c}:$ dos ; d et e : détails. 
Sur la face antérieure de la colonne, légèrement désaxé à gauche, un personnage nu, debout sur la base, est figuré de dos, plaqué contre le fût et les bras levés dans une attitude d'imploration. Il s'agit assurément d'un orant dont le corps musclé se confond presque avec le fût de la colonne.

L'objet est manifestement antique ${ }^{71}$ mais il ne peut guère être daté avec plus de précision que des trois premiers siècles de notre ère. Cependant, l'utilisation de la colonne "toscane " nous ferait pencher en faveur du $\mathrm{II}^{\mathrm{e}}$ ou du $\mathrm{III}^{\mathrm{e}}$ siècle.

Ce dieu stylite est d'origine inconnue mais son attribution au Massif central nous paraît raisonnable ${ }^{72}$.

L'iconographie de cette étrange divinité est unique à notre connaissance ct nous sommes bien en peine de l'identifier. Nu et imploré par un orant, il s'agit bien d'une divinité aux caractères monstrueux évidents. Bien que juchée sur une colonne, elle ne présente aucune parenté avec le Jupiter cavalier à l'anguipède. En revanche, les ailes remplaçant les bras évoquent un dieu-oiscau (ou une contamination, mal comprise, par les ailcrons de tête de Mercure?), un dieu de l'empyrée, un dicu céleste et non une divinité chthonienne.

La tête bouffie, au crâne rasé, aux arcades sourcilières accusées et aux larges oreilles, évoque le Mercure de Tongres qui porte, lui aussi, des cornes ou ailerons embryonnaires, mais également toutes les têtes " orientalisantes " à cirrus. L'attitude est ici très voisine de celle du dieu triphallique et si ce dernier caractère est absent chez le dieu stylite, il faut peut-être rapprocher du coq (ou de l'oiscau aux ailes déployées) du Mercure de Tongres la singulière métamorphose de ses bras. Après tout, l'oiseau de Tongres resscmble plus à un corbeau, au bec fort et à l'attitude rengorgéc des corvidés, qu'au coq "gaulois» cher à Mercure. Et l'on sait aussi que le corbeau est l'oiseau de Lug, dieu gaulois de la parentèle de Mercure qui en hérita bien des caractères ${ }^{73}$. Il n'est, par conséquent, pas impossible que ce dieu stylite inédit soit l'illustration d'une divinité indigène qui emprunte, elle aussi, quelques caractères à cette myriade de divinités indéfinies, qui ont dû varier dans le temps et dans l'espace et que l'on recouvre commodément, comme César le fit jadis, sous le vocable accueillant de Mercure.

71 Une fabrication récente est exclue par la nature mème de la corrosion. La patine est très peu épaisse ; clle est noire et quasi uniforme. Il s'agit probablement d'un oxyde de cuivre (ténorite) ou d'un sulfure de cuivre. Cette seconde hypothèse pourrait supposer l'inhumation ou plutôl l'immersion dans un milieu sulfureux et humide (zone volcanique active ou source sulfureuse). Renseignements communiqués par M ${ }^{\text {me }}$ B. Derion. Cette patine noire a élé partiellement recouverte, sur la moitié gauchc de l'objet, de taches épaisses de malachite (patine verte), dans laquelle sont incrustés quelques grains de quartz (et de calcite?) provenant du sédiment d'accueil.

Hormis la perte de l'aile gauche, l'objet est dans un bon état de conservation. La cassure est ancienne, probablement antique : la patine la recouvre. Le moignon brisé montre un métal peu homogène.

La fonte à la cire perdue a été faite sur un modèle maladroitement exéculé : la colonne conserve des traces de gouge ou d'ébauchoir ; des creux sont empâtés. Des facetles limées montrent la médiocrité de la finition. Malgré les apparences, il ne semble pas que l'on soit en présence d'une fonte avec utilisation d'un moule bivalve.

Des traces récentes de lime ont laissé à nu l'alliage jaune au dos du sujet. Sous la base, on observe également des traces de lime, anciennes (patinées) et récentes (métal à nu), un trou axial de fixation, un sccond trou tapissé de soudures récentes et des traces modernes de collage à la gommelaque.

L'originalité de l'iconographic plaide également dans le sens d'une création antique à motivation très complexe (H. : $9,3 \mathrm{~cm} ; 1 .: 2 \mathrm{~cm}$; ép. : $1,9 \mathrm{~cm}$ ).

72 Cet objet a été acquis sans origine, "entre 1940 et octobre 1948 ", par M. Paul Roudié, à la salle des ventes de limoges (Haute-Vienne). La statuette faisail partie d'un petit lot comprenant également un fac-similé moderne en bronze patiné de la dédicace de Matulinus Victorinus à Mercure Dumias (C.I.L., XIII, 1523), trouvece en 1874 au sanchuaire du sommet du Puy-de-Dôme, et conservéc au Musée Bargoin de Clermonl-Ferrand. Ce lol de deux pièces avail été soumis aux enchères, sans indication de provenance, au cours d'une vente de mobilier et d'objets domestiques modernes sans aucune valeur archéologique. La date et le lieu de la vente publique, l'association à la plaquette volive du sanctuaire du Dòme et le peu d'intérêt manifesté pour celle cuvre par l'ancien détenteur comme par le commissaircpriscur nous incitent à accepler l'hypothèse d'unc origine régionale de celle statuette dont on ne pouvait soupçonner alors l'intérêt archéologique.

73 P.-M. Duval, Les dieux de la Gaule, Paris, 1957, p. 67. 


\section{Les dieux slyliles el Grégoire de T'ours}

Cette statuette originale prend un relief tout particulier à la lecture d'un texte de Grégoire de Tours évoquant l'existence, à Brioude (Haute-Loire), d'un grand sanctuaire où figurait, juchée sur une colonne, l'image (simulacrum) de Mars et de Mercure ${ }^{74}$. P. Lambrechts, qui a exhumé ce texte pour faire référence aux divinités bicéphales, remarque l'emploi du singulier, d'une image unique pour évoquer tout à la fois Mars et Mercure ${ }^{75}$. Il note en particulier que les statues bifrons étaient juchées sur une haute colonne ou prolongées d'un corps en forme de gaine hermaïque ${ }^{76}$.

La statuette du dieu ailé à la colonne est un document tout à fait précieux illustrant ce texte de Grégoire de Tours. Comme le Mercure de Tongres, il évoque les simulacra, vues et combattues au $\mathrm{VI}^{\mathrm{e}}$ siècle encore par le saint évêque auvergnat: des statues de culte de divinités païennes placées sur de hautes colonnes isolées pour être présentées à la piété des fidèles au centre de sanctuaires importants ${ }^{77}$. Cette pratique ne devait pas être tout à fait courante à l'époque impériale, du moins dans les monuments en pierre ou en bronze, car les sources littéraires mais aussi les vestiges archéologiques l'eussent montrée plus souvent et de manière plus explicite. Cependant, ces divinités janiformes dont ccrtaines pouvaient présenter les mêmes attribuls que Mercure, ont pu être aisément attribuées par César au dieu romain du commerce, de la richesse et de l'invention technique.

Enfin, le dieu stylite et le Mercure de Tongres (qui, lui, a perdu son support), ne sont probablement pas la réduction fidèle de statues de culte mais plutôt, leur interprétation : la figure divine est manifestement privilégiée par rapport à son support et à l'orant de proportions beaucoup plus réduites.

\section{Mercure, divinité psychopompe}

Si les vertus prophylactiques, magiques et propitiatoires attribuées au phallus par les Anciens sont bien reconnues par tous ${ }^{78}$, le rôle funéraire du Mercure phallique gaulois n'apparaît pas aussi clairement. G. Picard attribue au Mercure triphallique de Tongres les caractères d'une "divinité psychopompe, et par cela mème ithyphallique $»^{79}$.

La statuette de Tongres fut, en effet, découverte dans une sépulture, comme ce fut le cas de beaucoup d'amulettes phalliques "classiques». Le Mercure phallique du Mas-d'Agenais provient également sinon d'une nécropole, du moins d'un lieu plus ou moins consacré aux habitants du monde souterrain, divinités chthoniennes ou âmes des morts. Il est donc bien tentant d'attribuer au Mercure phallique le rôle psychopompe que l'on reconnaît à l'Hermès gréco-romain, mais aussi à Priape ${ }^{80}$. Cependant, si les amulettes phalliques, et donc les Mercures phalliques, sont bien des talismans tout à la fois propitiatoires (puissance, fécondité, richesse, virilité surhumaine) et apotropaïques (protection contre le mauvais œil), si les amulettes phalliques sont fréquentes dans les tombes, il est absolument évident que l'on ne peut les considérer seulement comme des objets funéraires destinés à faciliter le passage du défunt dans l'au-delà mais qu'en revanche, elles étaient déposées dans les tombes pour

74 Gregorra de Tours, De passione et virtulibus sancli Iuliani Marlyris, $\mathrm{vi}^{\mathrm{c}}$ siecle, dans J. Zwick ER, Fontes Historiae Religionis Cellicae, p. 177. D'autres auleurs anciens citent des statues sur colonnes : Sulpice Sévèrs, Vita sancti Martini, dans J. Zwicker, op. cit., II, p. 121 ; VÉNanck Fortunat dans J. ZwICkrR, op. cil., II, p. 187.

75 P. LAmirechts, Nole sur un passage de Grégoire de Tours relalif à la religion gauloise, dans Lalomus, XIII, p. 207-217.

76 P. LambRichts, op. cil., p. 210-212.

77 Ne doit-on pas trouver là une justification des " ensembles ordonnés de poteaux "isolés dont on a retrouvé les trous sur l'aire sacrée de sanctuaires gaulois, comme à Gournaysur-Aronde (Oise)? (:f. J.-I. Bruxalx, Les sanchatures celliques et leurs dépôts, dans L'Art Celtique en Gaule, cat. d'expo., Marseille, Paris, Bordeaux, Dijon, 1983-1984, p. 81, dessin A. Rapin. J.-L. Brcvaux, P. Menifi. et F. Poplin, Gournay I, R.A.P., 1985, p. 91-92.

D'autres statuettes, mais elles sont très rares, sont susceptibles d'avoir été jadis placées sur une colonne. Ce pourrait être le cas, par exemple, du Mercure d'Ems (Autriche), réputé Mercure cavalier, dont l'altitude est très proche de celle du Mercure de Tongres et de notre dieu stylite. Stéphanie Boucure, Recherches, p. 154, pl. 73, no 354 .

78 Herter, dans R.E., s.v. phallos, col. 1683 sq.

79 G. PICARD, R.E.L., XXXI, 1953 (1954), p. 392.

80 F. Brisoit, Mars et Mercure, op. cit., p. 160 et note 104. 
continuer à protéger les morts comme, auparavant, elles l'avaient fait pour les vifs. L'usure des bélières montre sans équivoque que ces objets ont longuement servi aux vivants avant de trouver un usage définitif auprès des morts.

En tout état de cause, nous ne connaissons pas, pour cette époque, même dans les autres domaines de la civilisation matérielle (lampes à huile, vases de céramique, de verre ou de bronze, statuettes) d'objets qui soient spécifiques du culte funéraire, en Aquitaine du moins. Il n'y a pas en effet, d'urne cinéraire en terre ou en verre qui ne soit, à l'origine et avant tout, un vase culinaire dont on rencontre des fragments similaires dans les zones d'habitat.

Cette analyse du Mercure du Mas-d'Agenais, image géographiquement isolée qui associe de manière inhabituelle une figuration phallique à un buste divin d'apparence tout à fait classique, et la réflexion qu'elle nous conduit à avoir sur des figures apparentées, montrent bien, s'il était nécessaire, la complexité de la religion gauloise et la difficulté d'identifier des figurations rares dont l'iconographie incertaine est empruntée à des divinités multiples, nombreuses, mouvantes, aux attributs indéfinis ou polyvalents.

Nous avons observé le rôle sacré des représentations phalliques et cela plaide en faveur de l'hypothèse de L. Maurin qui voit dans les reliefs monstrucux de Saintonge, l'image de dieux-faces indigènes et plus encore, celle d'un Mercure gaulois, "divinité chthonienne et infernale, ... principe de fécondité et force créatrice, ... parèdre saintais de la déesse-mère, ... dieu local de la fécondité et de la richesse ${ }^{81}$, apparenté à Cernunnos, le porteur des bois de cerf, souvent tricéphale.

S'il manque encore de nombreux chaînons à là compréhension des divinités indigènes, très nombreuses, qui furent plus ou moins "interprétées " sous les traits et le nom du Mercure des Romains, la petite amulette du Mas-d'Agenais et la statuctte du dieu stylite se révèlent des documents particulièrement précicux dont on discutera longtemps encore l'interprétation. Ces objets nous ont offert cependant l'occasion de proposer une lecture peut-être plus logique, plus cohérente, d'un ensemble de documents abscons, faute d'être évoqués par les sources littéraires ou épigraphiques. Loin d'être des fantaisies de bronziers, ces statuettes s'inscrivent dans une série d'objets qui s'éclairent mutuellement. Elles témoignent de l'existence d'une iconographie religieuse "marginale " à la signification probablement évidente pour les populations gauloise ou gallo-romaine dont elle émane, signification complexe qu'il nous est bien difficile de cerner aujourd'hui.

Jacques SANTROT

N.B. - Je remercie toul particulierement MM. J.-J. IIatt, L. Maurin, J, Marcadé, I. Richard, J. Schwartz et. F. Tassaux qui ont bien voulu relire ce texte et me prodiguer leurs conseils.

81 L. Maunix, Saintes Antique, p. 241-242.
Les illustrations pholographiques sont de : J.-M. Arnaud, Musée d'Aquitaine, Bordeaux : fig. 1, al, b, c; 13 et 14. -. ch. Ilémon, Vusées départementaux de Loire-Allantique : fig. 2 et 3. - L. Maurin, Musée archéologique de Saintes : fig. 4 et 5. - G. Vienne, Musée archéologique de Saintes : fig. 6 et 7 . - Provinciaal Gallo-Romeins Museum Tongres (Belgique) : fig. 8. - Centre Jean Bćrard, Institut Français de Taples : fig. 9 .

Les dessins sont de : M.-II. Santrot : fig. 1 a2 et 10 . M.-H. et J. Santrot: fig. 11 . 